Pacific

Journal of

Mathematics

\title{
FOURIER TRANSFORMS \\ OF SEMISIMPLE ORBITAL INTEGRALS \\ ON THE LIE ALGEBRA OF SL 2
}

LOREN SPICE

Volume $254 \quad$ No. 2

December 2011 


\title{
FOURIER TRANSFORMS \\ OF SEMISIMPLE ORBITAL INTEGRALS \\ ON THE LIE ALGEBRA OF SL2
}

\author{
LOREN SPICE
}

The Harish-Chandra-Howe local character expansion expresses the characters of reductive, $p$-adic groups in terms of Fourier transforms of nilpotent orbital integrals on their Lie algebras, and Murnaghan-Kirillov theory expresses many characters of reductive, $p$-adic groups in terms of Fourier transforms of semisimple orbital integrals (also on their Lie algebras). In many cases, the evaluation of these Fourier transforms seems intractable, but for $\mathrm{SL}_{2}$, the nilpotent orbital integrals have already been computed. We compute Fourier transforms of semisimple orbital integrals using a variant of Huntsinger's integral formula and the theory of $p$-adic special functions.

$\begin{array}{ll}\text { 1. Introduction } & 407 \\ \text { 2. Notation } & 410 \\ \text { 3. Fields and algebras } & 412 \\ \text { 4. Tori and filtrations } & 413 \\ \text { 5. Orbital integrals } & 416 \\ \text { 6. Roots of unity and other constants } & 418 \\ \text { 7. Bessel functions } & 421 \\ \text { 8. A mock Fourier transform } & 427 \\ \text { 9. Split and unramified orbital integrals } & 432 \\ \text { 10. Ramified orbital integrals } & 435 \\ \text { 11. An integral formula } & 441 \\ \text { Acknowledgements } & 444 \\ \text { References } & 444\end{array}$

\section{Introduction}

1A. History. Harish-Chandra's $p$-adic Lefschetz principle suggests that results in real harmonic analysis should have analogues in $p$-adic harmonic analysis. This principle has had too many successes to list, but it is interesting that the paths to

The author was partially supported by NSF award DMS-0854897.

MSC2010: 22E35, 22E50.

Keywords: $p$-adic, orbital integral, special function. 
results in the Archimedean and non-Archimedean settings are often different. One striking manifestation of this is that the characters for the discrete series of real groups were found before the representations to which they were associated were constructed (see [Harish-Chandra 1966, Theorem 16; Schmid 1968, Theorem 4]), whereas, in the $p$-adic setting, we now have explicit constructions of many representations (see [Howe 1971; Corwin 1989; Moy 1986; Morris 1992; Bushnell and Kutzko 1993a; 1993b; 1994; Moy and Prasad 1994; Adler 1998; Yu 2001; Stevens 2008], among many others), but explicit character tables are still very rare.

This scarcity is of particular concern because, as suggested by Sally, it should be the case that "characters tell all" [Sally and Spice 2009, p. 104]. Note, for example, the recent work of Langlands [2011], which uses in a crucial way (see [ibid., Section 1.d]) the character formulae of [Sally and Shalika 1968] to show the existence of a transfer map dual to the transfer of stable characters, but only for $\mathrm{SL}_{2}$. It seems likely that one of the main obstacles to extending the results of [Langlands 2011] to other groups is the absence of explicit character formulae for them.

The good news here is that much is known about the behaviour of characters in general. For example, the Harish-Chandra-Howe local character expansion [Howe 1973; Harish-Chandra 1999; DeBacker 2002] and Murnaghan-Kirillov theory [Murnaghan 1995a; 1995b; 1996a; 1996b; 2000; Kim and Murnaghan 2003; 2006] give information about the asymptotics (near the identity element) of characters of $p$-adic groups in terms of Fourier transforms of orbital integrals (nilpotent or semisimple) on the Lie algebra, and many existing character formulae are stated in terms of such orbital integrals; see, for example, [DeBacker 1997, Theorem 5.3.2; Spice 2005, Theorems 6.6 and 7.18; Adler and Spice 2009, Theorem 7.1; DeBacker and Reeder 2009, Lemma 10.0.4]. See also [Adler and Spice 2009, Section 0.1] for a more exhaustive description of what is known in the supercuspidal case.

The bad news is that many applications require completely explicit character tables - in particular, the evaluation of Fourier transforms of orbital integrals when they appear — but Hales [1994] has shown that the orbital integrals may themselves be "nonelementary". This term has a technical meaning, but, for our purposes, it suffices to regard it informally as meaning "difficult to evaluate". (Note, though, that the asymptotic behaviour of orbital integrals "near $\infty$ " is understood in all cases; see [Waldspurger 1995, Proposition VIII.1].) Since $\mathrm{SL}_{2}$ is both simple enough for many explicit computations to be tractable (for example, the Fourier transforms of nilpotent orbital integrals have already been computed [DeBacker and Sally 2000, Appendix A.3-A.4]), and complicated enough for interesting phenomena to be apparent (for example, unlike $\mathrm{GL}_{2}$ and $\mathrm{PGL}_{2}$, it admits nonstable characters), it is a natural focus for our investigations.

Another perspective on the behaviour of characters in the range where MurnaghanKirillov theory holds is offered in [Corwin et al. 1995, Theorem 4.2(d); Takahashi 
2003, Proposition 2.9(2); 2005, Theorem 2.5], where explicit mention of orbital integrals is replaced (on the "bad shell" — see Section 10B) by arithmetically interesting sums, identified in [Takahashi 2003; 2005] as Kloosterman sums. In fact, exponential sums - specifically, Gauss sums - have long been observed in $p$-adic harmonic analysis; see, for example, [Corwin et al. 1995, Proposition 3.7; Waldspurger 1995, Section VIII.1; DeBacker 1997, p. 55; Shalika 2004, Section 1.3; Adler and Spice 2009, Section 5.2].

The work recorded here was carried out while preparing [Adler et al. 2011], which provides a proof of the aforementioned $\mathrm{SL}_{2}$ character formulae [Sally and Shalika 1968] by specialising the results of [Adler and Spice 2009; DeBacker and Reeder 2009]. As discussed above, these general results are stated in terms of Fourier transforms of orbital integrals (see Definition 5.5); so, in order to obtain completely explicit formulae, it was necessary to evaluate those Fourier transforms. The author of the present paper was surprised to discover that this latter evaluation reduced to the computation of Bessel functions (see Section 7 and Proposition 8.11). In retrospect, by the $p$-adic Lefschetz principle mentioned on the first page, it seems natural that the "special functions" described in [Sally and Taibleson 1966] will play some important role in $p$-adic harmonic analysis, since their classical analogues are so integral to real harmonic analysis (see, for just one example, [Gindikin and Karpelevič 1962, Theorem 2], where Harish-Chandra's $\boldsymbol{c}$-function is calculated in terms of $\Gamma$-functions). Relationships between a different sort of Bessel function and a different sort of orbital integral (adapted to the Jacquet-Ye relative trace formula) have already been demonstrated by Baruch [1997; 2001; $2003 ; 2004 ; 2005]$. We will investigate further applications of complex-valued $p$-adic special functions in future work.

See also [Cunningham and Gordon 2009, Section 4] for a motivic approach to the calculation of Fourier transforms of semisimple orbital integrals.

1B. Outline of the paper. We need a lot of notation in order to be completely explicit; we describe it in Sections 2-7. Specifically, Sections 2-4 describe the basic notation for working with groups over $p$-adic fields, adapted to the particular setting of the group $\mathrm{SL}_{2}$. Since our formulae will be written "torus-by-torus" (à la [Harish-Chandra 1970, Theorem 12]), we need to describe the tori in $\mathrm{SL}_{2}$. This can be done very concretely; see Definition 4.1.

In Section 5, we define the functions $\hat{\mu}_{X^{*}}^{G}$ (Fourier transforms of orbital integrals) that we want to compute as representing functions for certain invariant distributions on $\mathfrak{s l}_{2}$ (see Definition 5.5 and Notation 5.7). Since these functions are defined only up to scalar multiples, it is important to be aware of the normalisations involved in their construction. We specify the (Haar) measures that we are using in Definition 2.1 and Proposition 11.2. 
As mentioned in Section 1A, $p$-adic harmonic analysis tends to involve Gauss sums and other fourth roots of unity, and our calculations are no exception; we define and compare some of the relevant constants in Section 6. Finally, with these ingredients in place, we can follow [Sally and Taibleson 1966] in defining the Bessel functions that we will use to evaluate $\hat{\mu}_{X^{*}}^{G}$. Already, [Sally and Taibleson 1966] offers considerable information about the values of these functions, but we need to carry the calculations further, especially far from the identity (see Proposition 7.5) and on the "bad shell" (see Proposition 7.7), where (twisted) Kloosterman sums make an appearance.

In Section 8, we define a function $M_{X^{*}}^{G}$ (see Definition 8.4), which we will spend most of the rest of the paper computing. This is a reasonable focus because, once the computations are completed, Proposition 11.2 will show that we have actually been computing $\hat{\mu}_{X^{*}}^{G}$. The definition of $M_{X^{*}}^{G}$ involves a rather remarkable function $\varphi_{\theta}$ (see Definition 8.2 and Lemma 8.3); it seems likely that generalising our techniques will require understanding the proper replacement for $\varphi_{\theta}$.

Proposition 8.11 describes $M_{X^{*}}^{G}$ in terms of Bessel functions, and Proposition 8.13 uses Theorem 7.4 to describe their behaviour near 0 .

We now proceed according to the "type" of $X^{*}$ (as in Definition 4.4). The calculations when $X^{*}$ is split, and when it is unramified, are quite similar; we combine them in Section 9. We split into cases depending on whether the argument to $M_{X^{*}}^{G}$ is far from (as in Section 9A) or close to (as in Section 9B) zero; there are qualitative differences in the behaviour, as can be seen by comparing, for example, Theorems 9.5 and 9.7. When $X^{*}$ is ramified, it turns out that, in addition to the behaviour far from (as in Section 10A) and close to (as in Section 10C) zero, there is a third range of interest in the middle. This is the so called "bad shell" (see Section 10B), and it seems likely that the particularly complicated nature of the formulae here is a reflection of the "nonelementary" behaviour of orbital integrals (hence, by Murnaghan-Kirillov theory, also of characters) described in [Hales 1994].

Finally, we show in Section 11 that the function we have been evaluating actually does represent the desired distribution, that is, equals $\hat{\mu}_{X^{*}}^{G}$. (See Proposition 11.2.) We close with some observations (see Theorem 11.3) about the qualitative behaviour of orbital integrals that does not depend (much) on the "type" of $X^{*}$.

\section{Notation}

Suppose that $k$ is a nondiscrete, non-Archimedean local field. We do not make any assumptions on its characteristic, but we assume that its residual characteristic $p$ is not 2. (We occasionally cite [Shalika 2004], which works only with characteristic-0 fields, but we shall not use any results from there that require this restriction.) Let 
$R$ denote the ring of integers in $k, \wp$ the prime ideal of $R$, and ord the valuation on $k$ with value group $\mathbb{Z}$.

Let $\mathfrak{f}$ denote the residue field $R / \wp$ of $k$. Write $q=|\mathfrak{f}|$ for the number of elements in $\mathfrak{f}$, and put $|x|=q^{-\operatorname{ord}(x)}$ for $x \in k$. If $\alpha \in \mathbb{C}$, then write $\nu^{\alpha}$ for the (multiplicative) character $x \mapsto|x|^{\alpha}$ of $k^{\times}$.

Put $\boldsymbol{G}=\mathrm{SL}_{2}$ and $G=\boldsymbol{G}(k)$, and let $\mathfrak{g}$ and $\mathfrak{g}^{*}$ denote the Lie algebra and dual Lie algebra of $G$, respectively.

It is important for our calculations to be quite specific about the Haar measures that we are using. For convenience, we fix the ones used in [Sally and Taibleson 1966, p. 280].

Definition 2.1. Throughout, we shall use the (additive) Haar measure $\mathrm{d} x$ on $k$ that assigns measure 1 to $R$, and the associated (multiplicative) Haar measure $\mathrm{d}^{\times} x=$ $|x|^{-1} \mathrm{~d} x$ on $k^{\times}$that assigns measure $1-q^{-1}$ to $R^{\times}$. When convenient, we shall write $\mathrm{d} t$ instead of $\mathrm{d} x$.

Definition 2.2. If $\Phi$ is an (additive) character of $k$, then define $\Phi_{b}: x \mapsto \Phi(b x)$ for $b \in k$. The depth of $\Phi$ is

$$
\mathrm{d}(\Phi):= \begin{cases}\min \left\{i \in \mathbb{Z}: \Phi \text { is trivial on } \wp^{i+1}\right\} & \text { if } \Phi \text { is nontrivial, } \\ -\infty & \text { otherwise. }\end{cases}
$$

The depth of a character is related to what is often called its conductor by $\mathrm{d}(\Phi)=$ $\omega(\Phi)-1$ (in the notation of [Shalika 2004, Section 1.3]). We have that

$$
\mathrm{d}\left(\Phi_{b}\right)=\mathrm{d}(\Phi)-\operatorname{ord}(b) .
$$

The notion of depth and the symbol d will be used in multiple contexts (see Definition 4.9); we rely on the context to disambiguate them.

Notation 2.4. $\Phi$ is a nontrivial (additive) character of $k$.

One of the crucial tools of Harish-Chandra's approach to harmonic analysis is the reduction, whenever possible, of questions about a group to questions about its Lie algebra. The exponential map often allows one to effect this reduction, but, since it might converge only in a very small neighbourhood of 0 , we replace it with a "mock-exponential map" (see [Adler 1998, Section 1.5]) which has many of the same properties (see Lemma 2.6).

Definition 2.5. The Cayley map c $: k \backslash\{1\} \rightarrow k \backslash\{-1\}$ is defined by

$$
\mathrm{c}(X)=(1+X)(1-X)^{-1} \text { for } X \in k \backslash\{1\} .
$$

The Cayley function is available in many settings; we are using it only as a function defined almost everywhere on $k$. 


\section{Lemma 2.6.}

- The map c is a bijection.

- $\mathrm{c}(-X)=\mathrm{c}(X)^{-1}=\mathrm{c}^{-1}(X)$ for $X \in k \backslash\{ \pm 1\}$.

- The map c carries $\wp^{i}$ to $1+\wp^{i}$ for all $i \in \mathbb{Z}_{>0}$.

- In the notation of Definition 2.1, the pullback along $\mathrm{c}$ of the measure $\mathrm{d}^{\times} x$ on $1+\wp$ is the measure $\mathrm{d} x$ on $\wp$.

- If $X \in \wp^{i}$ and $Y \in \wp^{j}$, with $i, j \in \mathbb{Z}_{>0}$, then

$$
\mathrm{c}(X+Y) \equiv \mathrm{c}(X)+2 Y\left(\bmod 1+\wp^{n}\right),
$$

where $n=j+\min \{2 i, j\}$.

Proof. It is easy to check that $x \mapsto(1-x)(1+x)^{-1}$ is inverse to $\mathrm{c}$ and satisfies the desired equalities and check that $\mathrm{c}\left(\wp^{i}\right) \subseteq 1+\wp^{i}$ and $\mathrm{c}^{-1}\left(1+\wp^{i}\right) \subseteq \wp^{i}$. If $f \in C^{\infty}(1+\wp)$, then there is some $i \in \mathbb{Z}_{>0}$ such that $f \in C\left(1+\wp / 1+\wp^{i}\right)$. Since $\operatorname{meas}_{\mathrm{d} x}\left(\wp^{i}\right)=q^{-i}=\operatorname{meas}_{\mathrm{d}^{\times} x}\left(1+\wp^{i}\right)$, we see that

$$
\begin{aligned}
\int_{1+\wp} f(x) \mathrm{d}^{\times} x & =\sum_{x \in 1+\wp / 1+\wp^{i}} f(x) \operatorname{meas}_{\mathrm{d}^{\times} x}\left(1+\wp^{i}\right) \\
& =\sum_{x \in \wp / \wp^{i}}(f \circ \mathrm{c})(x) q^{-i} \operatorname{meas}_{\mathrm{d} x}\left(\wp^{i}\right)=\int_{\wp}(f \circ \mathrm{c})(x) \mathrm{d} x .
\end{aligned}
$$

Finally, under the stated conditions on $X$ and $Y$,

$$
\begin{aligned}
(\mathrm{c}(X)+2 Y)(1-(X+Y)) & =\mathrm{c}(X) \cdot(1-X)+Y(2(1-(X+Y))-\mathrm{c}(X)) \\
& =(1+X+Y)+Y((1-2 X-\mathrm{c}(X))-2 Y) .
\end{aligned}
$$

Since $c(X)=1+2 X(1-X)^{-1}$, we have that $1-2 X-\mathrm{c}(X) \in \wp^{2 i}$. The result follows.

\section{Fields and algebras}

Definition 3.1. For $\theta \in k^{\times}$, write $k_{\theta}$ for the $k$-algebra that is $k \oplus k$ (as a vector space), equipped with multiplication $(a, b) \cdot(c, d)=(a c+b d \theta, a d+b c)$. Write $\sqrt{\theta}$ for the element $(0,1) \in k_{\theta}$, so that $(a, b)=a+b \sqrt{\theta}$.

We also use the notation $\sqrt{\theta}$ for a matrix (see Definition 4.1 ); we shall rely on context to make the meaning clear.

If $\theta \notin\left(k^{\times}\right)^{2}$, then $k_{\theta}$ is isomorphic to $k(\sqrt{\theta})$ (as $k$-algebras) via the map $(a, b) \mapsto$ $a+b \sqrt{\theta}$, and we shall not distinguish between them.

If $\theta=x^{2}$, with $x \in k$, then $k_{\theta}$ is isomorphic to $k \oplus k$ (as $k$-algebras) via the map $(a, b) \mapsto(a+b x, a-b x)$. 
Definition 3.2. Define

$$
\begin{aligned}
N_{\theta}(a+b \sqrt{\theta}) & =a^{2}-b^{2} \theta, \quad \operatorname{tr}_{\theta}(a+b \sqrt{\theta})=2 a, \\
\operatorname{Re}_{\theta}(a+b \sqrt{\theta}) & =a, \quad \operatorname{Im}_{\theta}(a+b \sqrt{\theta})=b, \\
\operatorname{ord}_{\theta}(a+b \sqrt{\theta}) & =\frac{1}{2} \operatorname{ord}\left(N_{\theta}(a+b \sqrt{\theta})\right)
\end{aligned}
$$

for $a+b \sqrt{\theta} \in k_{\theta}$. Write $C_{\theta}=\operatorname{ker} N_{\theta}$ and $V_{\theta}=\operatorname{ker} \operatorname{tr}_{\theta}$, and let $\operatorname{sgn}_{\theta}$ be the unique (multiplicative) character of $k^{\times}$with kernel precisely $N_{\theta}\left(k_{\theta}^{\times}\right)$.

If $\theta \notin\left(k^{\times}\right)^{2}$, then $N_{\theta}$ and $\operatorname{tr}_{\theta}$ are the usual norm and trace maps associated to the quadratic extension of fields $k_{\theta} / k$, and $\operatorname{ord}_{\theta}$ is the valuation on $k_{\theta}$ extending ord. In any case, $k_{\theta}^{\times}=\left\{z \in k_{\theta}: N_{\theta}(z) \neq 0\right\}$.

We can describe the signum character explicitly by

$$
\begin{aligned}
& \operatorname{sgn}_{\theta}(x)= \begin{cases}1 & \theta \text { split, } \\
(-1)^{\operatorname{ord}(x)} & \theta \text { unramified, }\end{cases} \\
& \left\{\begin{array}{l}
\operatorname{sgn}_{\theta}(\theta)=\operatorname{sgn}_{\mathfrak{f}}(-1) \\
\operatorname{sgn}_{\theta}(x)=\operatorname{sgn}_{\mathfrak{f}}(\bar{x}) \quad \text { for } x \in R^{\times},
\end{array}\right.
\end{aligned}
$$

where $\operatorname{sgn}_{\mathfrak{f}}$ is the quadratic character of $\mathfrak{f}^{\times}$and $x \mapsto \bar{x}$ the reduction map $R \rightarrow \mathfrak{f}$.

\section{Tori and filtrations}

We begin by defining a few model tori.

Definition 4.1. For $\theta \in k$, put

Then

$$
\boldsymbol{T}_{\theta}=\left\{\left(\begin{array}{cc}
a & b \\
b \theta & a
\end{array}\right): a^{2}-b^{2} \theta=1\right\} .
$$

$$
\mathfrak{t}_{\theta}:=\operatorname{Lie}\left(\boldsymbol{T}_{\theta}\right)=\left\{\left(\begin{array}{cc}
0 & b \\
b \theta & 0
\end{array}\right)\right\}
$$

Write $\sqrt{\theta}$ for the element

$$
\left(\begin{array}{ll}
0 & 1 \\
\theta & 0
\end{array}\right)
$$

so that $\mathfrak{t}_{\theta}=\operatorname{Span}_{k} \sqrt{\theta}$. Call a maximal $k$-torus in $\boldsymbol{G}$ standard exactly when it is of the form $\boldsymbol{T}_{\theta}$ for some $\theta \in k$.

We also denote by $\sqrt{\theta}$ a specific element of an extension of $k$ (see Definition 4.1); we shall rely on context to make the meaning clear.

Remark 4.2. The group $T_{\theta}$ is isomorphic to $C_{\theta}=\operatorname{ker} N_{\theta}$ and the Lie algebra $\mathfrak{t}_{\theta}$ to $V_{\theta}=\operatorname{ker} \operatorname{tr}_{\theta}$ in each case via the map

$$
\left(\begin{array}{cc}
a & b \\
b \theta & a
\end{array}\right) \mapsto(a, b)
$$


We shall use the terms "split", "unramified", and "ramified" in many different contexts.

Remark 4.3. If $\boldsymbol{T}$ is a maximal $k$-torus in $\boldsymbol{G}$ and $\mathfrak{t}=\operatorname{Lie}(T)$, then we shall identify $\mathfrak{t}$ (respectively, $\mathfrak{t}^{*}$ ) with the spaces of fixed points for the adjoint (respectively, coadjoint) action on $\mathfrak{g}$ (respectively, $\mathfrak{g}^{*}$ ). By abuse of language, we shall sometimes say that $X^{*} \in \mathfrak{g}^{*}$ or $Y \in \mathfrak{g}$ lies in, or belongs to, the torus $\boldsymbol{T}$ to mean that $X^{*} \in \mathfrak{t}^{*}$ and $Y \in \mathfrak{t}$; equivalently, that $C_{G}\left(X^{*}\right)=\boldsymbol{T}=C_{G}(Y)$. In particular, " $X^{*}$ and $Y$ belong to a common torus" is shorthand for " $C_{G}\left(X^{*}\right)=C_{G}(Y)$ ".

Definition 4.4. A maximal $k$-torus in $\boldsymbol{G}$ is called (un)ramified if it is elliptic and splits over an (un)ramified extension of $k$. An element $\theta \in k$ is called split, unramified, or ramified if $\boldsymbol{T}_{\theta}$ has that property. A regular, semisimple element of $\mathfrak{g}$ or $\mathfrak{g}^{*}$ is called split, unramified, or ramified if the torus to which it belongs has that property.

Remark 4.5. To be explicit, squares in $k^{\times}$are split, and a nonsquare $\theta \in k$ is unramified or ramified if $\max \left\{\operatorname{ord}\left(x^{2} \theta\right): x \in k\right\}$ is even or odd, respectively.

Notation 4.6. If $\boldsymbol{T}$ is a maximal $k$-torus in $\boldsymbol{G}$ with $T=\boldsymbol{T}(k)$, then write $W(\boldsymbol{G}, \boldsymbol{T})=$ $N_{\boldsymbol{G}}(\boldsymbol{T}) / \boldsymbol{T}$ for the absolute and $W(G, T)=N_{G}(T) / T$ for the relative Weyl group of $\boldsymbol{T}$ in $\boldsymbol{G}$.

Every maximal $k$-torus in $\boldsymbol{G}$ is $G$-conjugate to some $\boldsymbol{T}_{\theta}$. (See, for example, [DeBacker and Sally 2000, Section A.2].) In particular,

$$
\operatorname{Int}\left(\begin{array}{cc}
1 & 1 \\
-1 / 2 & 1 / 2
\end{array}\right)\left\{\left(\begin{array}{ll}
a & 0 \\
0 & d
\end{array}\right): a d=1\right\}=\boldsymbol{T}_{1} .
$$

Remark 4.7. For all $\theta \in k$, the group $W\left(\boldsymbol{G}, \boldsymbol{T}_{\theta}\right)$ has order 2, with the nontrivial element acting on $\boldsymbol{T}_{\theta}$ by inversion. If $\operatorname{sgn}_{\theta}(-1)=1$ (in particular, if $\theta$ is split or unramified), say, with $N_{\theta}(a+b \sqrt{\theta})=-1$, then $W\left(G, T_{\theta}\right)$ also has order 2, with the nontrivial element represented by

$$
\left(\begin{array}{cc}
a & b \\
-b \theta & -a
\end{array}\right)
$$

If $\theta=1$, then we may take $(a, b)=(0,1)$ to recover the familiar Weyl group element. Otherwise (that is, if $\left.\operatorname{sgn}_{\theta}(-1)=-1\right), W\left(G, T_{\theta}\right)$ is trivial.

The concept of stable conjugacy was introduced by Langlands [1979, pp. 2-3] as part of the foundation of the Langlands conjectures.

\section{Definition 4.8. Two}

- maximal $k$-tori $\boldsymbol{T}_{i}$ in $\boldsymbol{G}$,

- regular semisimple elements $X_{i}^{*} \in \mathfrak{g}^{*}$, or

- regular semisimple elements $Y_{i} \in \mathfrak{g}$, 
with $i=1,2$, are called stably conjugate exactly when there are a field extension $E / k$ and an element $g \in \boldsymbol{G}(E)$ such that

- $\operatorname{Int}(g) T_{1}=T_{2}$,

- $\operatorname{Ad}^{*}(g) X_{1}^{*}=X_{2}^{*}$, or

- $\operatorname{Ad}(g) X_{1}=X_{2}$,

where $T_{i}=\boldsymbol{T}_{i}(k)$ for $i=1,2$. If the conjugacy can be carried out without passing to an extension field (that is, if we may take $g \in G$ ), then we will sometimes emphasise this by saying that the tori or elements are rationally conjugate.

The Zariski-density of $T_{i}$ in $\boldsymbol{T}_{i}$ implies that $\operatorname{Int}(g) \boldsymbol{T}_{1}=\boldsymbol{T}_{2}$, but that this a strictly weaker condition; indeed, given any two maximal tori, there is an element $g$, defined over some extension field of $k$, satisfying this condition. In our special case (of $\boldsymbol{G}=\mathrm{SL}_{2}$ ), two tori or elements are stably conjugate if and only if they are conjugate in $\mathrm{GL}_{2}(k)$.

More concretely, two tori $\boldsymbol{T}_{\theta}$ and $\boldsymbol{T}_{\theta^{\prime}}$ are stably conjugate if and only if $\theta \equiv$ $\theta^{\prime}\left(\bmod \left(k^{\times}\right)^{2}\right)$. The stable conjugacy class of the split torus $\boldsymbol{T}_{1}$ is also a rational conjugacy class.

Suppose that $\epsilon$ is an unramified and $\varpi$ a ramified, nonsquare. Then the stable conjugacy class of $\boldsymbol{T}_{\epsilon}$ splits into 2 rational conjugacy classes, represented by $\boldsymbol{T}_{\epsilon}$ and $\boldsymbol{T}_{\varpi^{2} \epsilon}$. The stable conjugacy class of $\boldsymbol{T}_{\varpi}$ is also a rational conjugacy class if $\operatorname{sgn}_{\varpi}(-1)=-1$, but it splits into 2 rational conjugacy classes, represented by $\boldsymbol{T}_{\varpi}$ and $\boldsymbol{T}_{\epsilon^{2} \varpi}$, if $\operatorname{sgn}_{\varpi}(-1)=1$.

We also need filtrations on the Lie algebra and dual Lie algebra of a torus. These definitions are standard (see, for example, [Adler 1998, Section 1.4]) and can be made in far more generality (see [Moy and Prasad 1994, Section 3; 1996, Section 3.3]); we give only simple definitions adapted to $\boldsymbol{G}=\mathrm{SL}_{2}$.

Definition 4.9. Let $\boldsymbol{T}$ be a maximal $k$-torus in $\boldsymbol{G}$. Recall that $\boldsymbol{T}$ is $G$-conjugate to $\boldsymbol{T}_{\theta}$ for some $\theta \in k$, so that $\mathfrak{t}=\operatorname{Lie}(T)$ is isomorphic to $V_{\theta}=\operatorname{ker} \operatorname{tr}_{\theta} \subseteq k_{\theta}$. For $r \in \mathbb{R}$, write $\mathfrak{t}_{r}$ for the preimage of $\left\{Y \in V_{\theta}: \operatorname{ord}_{\theta}(Y) \geq r\right\}$ and $\mathfrak{t}_{r+}$ for the preimage of $\left\{Y \in V_{\theta}: \operatorname{ord}_{\theta}(Y)>r\right\} ;$ then write $\mathfrak{t}_{r}^{*}=\left\{X^{*} \in \mathfrak{t}^{*}: \Phi\left(\left\langle X^{*}, Y\right\rangle\right)=1\right.$ for all $\left.Y \in \mathfrak{t}_{(-r)+}\right\}$ (where $\Phi$ is the additive character of Notation 2.4).

If $X^{*} \in \mathfrak{t}^{*}$ and $Y \in \mathfrak{t}$, then define $\mathrm{d}\left(X^{*}\right)=\max \left\{r \in \mathbb{R}: X^{*} \in \mathfrak{t}_{r}^{*}\right\}$ and $\mathrm{d}(Y)=$ $\max \left\{r \in \mathbb{R}: Y \in \mathfrak{t}_{r}\right\}$.

One can define a notion of depth in more generality (see, for example, [Adler and DeBacker 2002, Section 3.3 and Example 3.4.6; Kim and Murnaghan 2003, Section 2.1 and Lemma 2.1.5]), but we only need the special case above. (The only remaining case to consider for $\mathfrak{g}=\mathfrak{s l}_{2}(k)$ is the depth of a nilpotent element, which is $\infty$.) 


\section{Orbital integrals}

Our goal in this paper is to compute Fourier transforms of regular, semisimple orbital integrals on $\mathfrak{g}$ (see Definition 5.5 below). Since the Fourier transforms of nilpotent orbital integrals were computed in [DeBacker and Sally 2000, Appen$\operatorname{dix} \mathrm{A}]$, this covers all Fourier transforms of orbital integrals on $\mathfrak{g}$ (for our particular case $\boldsymbol{G}=\mathrm{SL}_{2}$ ). The case of orbital integrals on $G$ was discussed in [Sally and Shalika 1984], as the culmination of the series of papers that began with [Sally and Shalika 1968; 1969].

We begin by choosing a representative for the regular, semisimple orbit of interest. By Section 4, we may choose this representative in a standard torus (in the sense of Definition 4.1).

Notation 5.1. $\beta, \theta \in k^{\times}$, and $X^{*}=\beta \cdot \sqrt{\theta} \in \mathfrak{t}_{\theta}^{*}$.

Here, we are implicitly using the identification of $\mathfrak{t}_{\theta}$ with $\mathfrak{t}_{\theta}^{*}$ via the trace form; what we really mean is that $\left\langle X^{*}, Y\right\rangle=\operatorname{tr} \beta \cdot \sqrt{\theta} \cdot Y$ for $Y \in \mathfrak{t}_{\theta}$, where $\langle\cdot, \cdot\rangle$ is the usual pairing between $\mathfrak{t}_{\theta}^{*}$ and $\mathfrak{t}_{\theta}$.

As in Definition 2.2, we may define a new character $\Phi_{\beta}$ of $k$, which we use often in our calculations.

Notation 5.2. $-r=\mathrm{d}\left(X^{*}\right), \Phi^{\prime}=\Phi_{\beta}$, and $r^{\prime}=\mathrm{d}\left(\Phi^{\prime}\right)$.

By Definition $4.9, Y \mapsto \Phi\left(\left\langle X^{*}, Y\right\rangle\right)$ is trivial on $\left(\mathfrak{t}_{\theta}\right)_{r+}$, but not on $\left(\mathfrak{t}_{\theta}\right)_{r}$. Therefore, $r^{\prime}=r+\frac{1}{2} \operatorname{ord}(\theta)$.

Since $C_{G}\left(X^{*}\right)=T_{\theta}$ is Abelian, it is unimodular, so there exists a measure on $G / C_{G}\left(X^{*}\right)$ invariant under the action of $G$ by left translation.

Notation 5.3. Let $\mathrm{d} \dot{g}$ be a translation-invariant measure on $G / C_{G}\left(X^{*}\right)$.

Since the orbit, $O_{X^{*}}^{G}$, of $X^{*}$ under the coadjoint action of $G$ is isomorphic as a $G$-set to $G / C_{G}\left(X^{*}\right)$, we could transport to it the measure on the latter space; but we do not find it convenient to do so.

Since $X^{*}$ is semisimple, $O_{X^{*}}^{G}$ is closed in $\mathfrak{g}^{*}$; see, for example, [Tauvel and $\mathrm{Yu}$ 2005, Proposition 34.3.2]. Therefore, the restriction to $\mathcal{O}_{X^{*}}^{G}$ of a locally constant, compactly supported function on $\mathfrak{g}^{*}$ remains locally constant and compactly supported, so that the following definition makes sense.

Definition 5.4. The orbital integral of $X^{*}$ is the distribution $\mu_{X^{*}}^{G}$ on $\mathfrak{g}^{*}$ defined by

$$
\mu_{X^{*}}^{G}\left(f^{*}\right)=\int_{G / C_{G}\left(X^{*}\right)} f^{*}\left(\operatorname{Ad}^{*}(g) X^{*}\right) \mathrm{d} \dot{g} \quad \text { for all } f^{*} \in C_{c}^{\infty}\left(\mathfrak{g}^{*}\right) .
$$

We are interested in the Fourier transform of $\mu_{X^{*}}^{G}$. The definition of the Fourier transform (of distributions or of functions) requires, in addition to a choice of additive character (see Notation 2.4), also a choice of Haar measure $\mathrm{d} Y$ on $\mathfrak{g}^{*}$; but 
we shall build this choice into our representing function (see Notation 5.7), so that it will not show up in our final answer.

Definition 5.5. The Fourier transform of the orbital integral of $X^{*}$ is the distribution $\hat{\mu}_{X^{*}}^{G}$ on $\mathfrak{g}$ defined for all $f \in C_{c}^{\infty}(\mathfrak{g})$ by

$$
\hat{\mu}_{X^{*}}^{G}(f)=\mu_{X^{*}}^{G}(\hat{f}),
$$

where

$$
\hat{f}\left(Y^{*}\right)=\int_{\mathfrak{g}} f(Y) \Phi\left(\left\langle Y^{*}, Y\right\rangle\right) \mathrm{d} Y \quad \text { for all } Y^{*} \in \mathfrak{g}^{*} .
$$

It is a result of Harish-Chandra [1999, Theorem 1.1] that $\hat{\mu}_{X^{*}}^{G}$ is representable on $\mathfrak{g}$, that is, there exists a locally integrable function $F$ on $\mathfrak{g}$ such that

$$
\hat{\mu}_{X^{*}}^{G}(f)=\int_{G} f(Y) F(Y) \mathrm{d} Y \quad \text { for all } f \in C_{c}^{\infty}(\mathfrak{g}) .
$$

One can say more about the behaviour and asymptotics of the function $F$. For example, it blows up as $Y$ approaches 0 , but its blow-up is controlled by a power of a discriminant function.

Definition 5.6. The Weyl discriminant on $\mathfrak{g}$ is the function $D_{\mathfrak{g}}: \mathfrak{g} \rightarrow \mathbb{C}$ such that, for all $Y \in \mathfrak{g}, D_{\mathfrak{g}}(Y)$ is the coefficient of the degree-1 term in the characteristic polynomial of $\operatorname{ad}(Y)$. Concretely,

$$
D_{\mathfrak{g}}\left(\begin{array}{cc}
a & b \\
c & -a
\end{array}\right)=4\left(a^{2}+b c\right) .
$$

Our main interest, however, is in the restriction of the function $F$ above to the set $\mathfrak{g}^{\text {rss }}$ of regular, semisimple elements, where it is locally constant.

Notation 5.7. By abuse of notation, write again $\hat{\mu}_{X^{*}}^{G}$ for the function that represents the restriction to $\mathfrak{g}^{\text {rss }}$ of $\hat{\mu}_{X^{*}}^{G}$.

When we refer to the computation of the Fourier transform of an orbital integral, it is actually the (scalar) function of Notation 5.7 that we are trying to compute. The main tool in this direction is a general integral formula of Huntsinger (see [Adler and DeBacker 2004, Theorem A.1.2]), but we find it easier to evaluate an integral adapted to our current setting (see Definition 8.4). The computation of this integral will occupy most of the paper; we finally prove it actually represents the distribution $\hat{\mu}_{X^{*}}^{G}$ in Proposition 11.2.

Finally, we fix an element at which to evaluate the functions of interest. Since $\hat{\mu}_{X^{*}}^{G}$, as just defined, and $M_{X^{*}}^{G}$ in Definition 8.4 are $G$-invariant functions on $\mathfrak{g}^{\text {rss }}$, we may again consider only elements of standard tori.

Notation 5.8. $s, \theta^{\prime} \in k^{\times}$, and $Y=s \cdot \sqrt{\theta^{\prime}} \in \mathfrak{t}_{\theta^{\prime}}$. 
We phrase our computations in terms of the values of two "basic" functions at $Y$. Lemma 5.9. $\mathrm{d}(Y)=\frac{1}{2} \operatorname{ord}\left(s^{2} \theta^{\prime}\right)$ and $D_{\mathfrak{g}}(Y)=4 s^{2} \theta^{\prime}$.

Proof. This is a straightforward consequence of Definitions 4.9 and 5.6.

\section{Roots of unity and other constants}

The computation of Fourier transforms of orbital integrals on $\mathfrak{g}$ via MurnaghanKirillov theory [Murnaghan 1995a; Kim and Murnaghan 2003; 2006; Adler and DeBacker 2004; Adler and Spice 2009] and also of the values near the identity of characters of $G$ (see [Sally and Shalika 1968; Adler et al. 2011]) involves a somewhat bewildering array of 4-th roots of unity, for each of which there is a variety of notation available. All of these can be expressed in terms of a single "basic" quantity, the Gauss sum, denoted by $G(\Phi)$ in [Shalika 2004, Lemma 1.3.2]. The definition there implicitly depends on a choice of uniformiser, denoted there by $\pi$. Although the choice is arbitrary, for later convenience we denote it by $-\varpi$. Recall from Notation 2.4 that $\Phi$ is a nontrivial (additive) character of $k$.

Definition 6.1. If $\varpi$ is a uniformiser of $k$, then

$$
G_{\varpi}(\Phi):=q^{-1 / 2} \sum_{X \in R / \wp} \Phi_{(-\varpi)^{\mathrm{d}(\Phi)}}\left(X^{2}\right) .
$$

It is possible to compute these values exactly (see, for example, [Lidl and Niederreiter 1997, Theorem 5.15]), but we only require a few transformation laws.

Lemma 6.2. If $\varpi$ is a uniformiser of $k$, then

$$
\begin{aligned}
& G_{b \varpi}(\Phi)=\operatorname{sgn}_{\varpi}(b)^{\mathrm{d}(\Phi)} G_{\varpi}(\Phi) \quad \text { for } b \in R^{\times}, \\
& G_{\varpi}\left(\Phi_{b}\right)=\operatorname{sgn}_{\varpi}(b) G_{\varpi}(\Phi) \quad \text { for } b \in k^{\times}, \\
& G_{\varpi}(\Phi)^{2}=\operatorname{sgn}_{\varpi}(-1) \text {, } \\
& G_{\varpi}(\Phi)=q^{-1 / 2} \operatorname{sgn}_{\varpi}(-1)^{\mathrm{d}(\Phi)} \sum_{X \in \mathfrak{f}^{\times}} \bar{\Phi}(X) \operatorname{sgn}_{\mathfrak{f}}(X),
\end{aligned}
$$

where $\operatorname{sgn}_{\mathfrak{f}}$ is the quadratic character of $\mathfrak{f}^{\times}$, and $\bar{\Phi}$ the (additive) character of $\mathfrak{f}=R / \wp$ arising from the restriction to $R$ of the depth-0 character $\Phi_{\varpi^{\mathrm{d}(\Phi)}}$ of $k$. Proof. Since $\sum_{X \in \mathfrak{f}} \bar{\Phi}(X)=0$, we have that

$$
\begin{aligned}
\sum_{X \in \mathfrak{f}^{\times}} \bar{\Phi}(X) \operatorname{sgn}_{\mathfrak{f}}(X) & =\bar{\Phi}(0)+\sum_{X \in \mathfrak{f}^{\times}} \bar{\Phi}(X)\left(1+\operatorname{sgn}_{\mathfrak{f}}(X)\right) \\
& =\bar{\Phi}(0)+2 \sum_{X \in\left(\mathfrak{f}^{\times}\right)^{2}} \bar{\Phi}(X)=\sum_{X \in \mathfrak{f}} \bar{\Phi}\left(X^{2}\right)=q^{1 / 2} G_{\varpi}\left(\Phi_{(-1)^{\mathrm{d}(\Phi)}}\right) .
\end{aligned}
$$

In other words,

$$
G_{\varpi}\left(\Phi_{(-1)^{\mathrm{d}(\Phi)}}\right)=q^{-1 / 2} G\left(\operatorname{sgn}_{\mathfrak{f}}, \bar{\Phi}\right),
$$


where the notation on the right is as in [Lidl and Niederreiter 1997, Section 5.2] (except that their $\psi$ is our $\operatorname{sgn}_{\mathfrak{f}}$, the quadratic character of $\mathfrak{f}^{\times}$, and their $\chi$ is our $\left.\bar{\Phi}\right)$. The third equality, and the second equality for $b \in R^{\times}$, now follow from [ibid., Theorem 5.12]. The first equality follows from the second since $G_{b \varpi}(\Phi)=G_{\varpi}\left(\Phi_{b^{\mathrm{d}(\Phi)}}\right)$. Taking $b=(-1)^{\mathrm{d}(\Phi)}$ and combining with $(*)$ gives the fourth equality. Finally, by definition, $G_{\varpi}\left(\Phi_{(-\varpi)^{n}}\right)=G_{\varpi}(\Phi)=\operatorname{sgn}_{\varpi}(-\varpi)^{n} G_{\varpi}(\Phi)$ for all $n \in \mathbb{Z}$.

By Proposition 8.11 and Theorem 7.4, our calculations will involve the $\Gamma$-factors defined in [Sally and Taibleson 1966, Section 3]. The factor $\Gamma\left(v^{1 / 2} \operatorname{sgn}_{\varpi}\right)$ is of particular interest. By [ibid., Theorem 3.1(iii)], $\Gamma\left(v^{1 / 2} \operatorname{sgn}_{\varpi}\right)^{2}=\operatorname{sgn}_{\varpi}(-1)$, so by Lemma 6.2, $\Gamma\left(v^{1 / 2} \operatorname{sgn}_{\varpi}\right)= \pm G_{\varpi}(\Phi)$. It will be useful to identify the sign.

Lemma 6.3. If $\varpi$ is a uniformiser of $k$, then

$$
\Gamma\left(v^{1 / 2} \operatorname{sgn}_{\varpi}\right)=\operatorname{sgn}_{\varpi}(-1)^{\mathrm{d}(\Phi)+1} G_{\varpi}(\Phi) .
$$

Proof. Write $\bar{\Phi}=\Phi_{\varpi^{\mathrm{d}(\Phi)}}$; this is a depth-0 character of $k$. The definitions of [Sally and Taibleson 1966] depend on a depth-(-1) additive character $\chi$; we take it to be $\bar{\Phi}_{\varpi}$. The definition of $\Gamma\left(v^{1 / 2} \operatorname{sgn}_{\varpi}\right)$ involves a principal-value integral (see Definition 8.4), but, as pointed out in the proof of [Sally and Taibleson 1966, Theorem 3.1], by [ibid., Lemma 3.1] and (3.4) it simplifies to

$$
\begin{aligned}
\Gamma\left(v^{1 / 2} \operatorname{sgn}_{\varpi}\right) & =\int_{\operatorname{ord}(x)=-1} \bar{\Phi}_{\varpi}(x)|x|^{1 / 2} \operatorname{sgn}_{\varpi}(x) \mathrm{d}^{\times} x \\
& =\int_{R^{\times}} \bar{\Phi}_{\varpi}\left(\varpi^{-1} x\right)\left|\varpi^{-1} x\right|^{1 / 2} \operatorname{sgn}_{\varpi}\left(\varpi^{-1} x\right) \mathrm{d}^{\times} x \\
& =q^{1 / 2} \operatorname{sgn}_{\varpi}(-1) \operatorname{meas}_{\mathrm{d}^{\times} x}(1+\wp) \sum_{x \in R^{\times} / 1+\wp} \bar{\Phi}(x) \operatorname{sgn}_{\mathfrak{f}}(x),
\end{aligned}
$$

where $\mathrm{d}^{\times} x$ is the Haar measure on $k^{\times}$giving $R^{\times}$measure $1-q^{-1}$ (see Definition 2.1). Since meas $_{\mathrm{d}^{\times} x}(1+\wp)=q^{-1}$, the result now follows from Lemma 6.2.

We will also need some constants associated to specific elements.

Waldspurger [1995, Proposition VIII.1] describes the "behaviour at $\infty$ " of Fourier transforms of semisimple orbital integrals on general reductive, $p$-adic Lie algebras. His description involves a 4-th root of unity $\gamma_{\psi}\left(X^{*}, Y\right)$ (see [ibid., p. 79]); since his $\psi$ is our $\Phi$ (Notation 2.4), we denote it by $\gamma_{\Phi}\left(X^{*}, Y\right)$. See Theorem 11.3 for our quantitative analogues (for the special case of $\mathfrak{s l}_{2}$ ) of his result.

We would like to avoid choosing "standard" representatives for $k^{\times} /\left(k^{\times}\right)^{2}$ (see Remark 6.9), but doing this is notationally unwieldy. Although our proofs will make use of these choices, none of the statements of the main results (except Theorems 10.8 and 10.9, via Remark 10.7) relies on them.

Notation 6.4. Let $\epsilon$ be a lift to $R^{\times}$of a nonsquare in $\mathfrak{f}^{\times}$and $\varpi$ a uniformiser of $k$. 
Definition 6.5. Recall Notations 5.1 and 5.8. If $X^{*}$ and $Y$ lie in stably conjugate tori, so that $\theta \equiv \theta^{\prime}\left(\bmod \left(k^{\times}\right)^{2}\right)$, then

$$
\gamma_{\Phi}\left(X^{*}, Y\right)= \begin{cases}1 & \theta \equiv 1, \\ \gamma_{\mathrm{un}}(s) & \theta \equiv \epsilon, \\ \gamma_{\mathrm{ram}}(s) & \theta \equiv \varpi, \\ -\gamma_{\mathrm{un}}(s) \gamma_{\mathrm{ram}}(s) & \theta \equiv \epsilon \varpi,\end{cases}
$$

where all congruences are taken modulo $\left(k^{\times}\right)^{2}$ and where

$$
\gamma_{\mathrm{un}}(s):=(-1)^{r^{\prime}+1} \operatorname{sgn}_{\epsilon}(s) \quad \text { and } \quad \gamma_{\mathrm{ram}}(s):=\operatorname{sgn}_{\varpi}(-s) G_{\varpi}\left(\Phi^{\prime}\right)
$$

(with notation as in Notation 5.2 and Definition 6.1). It simplifies our notation considerably also to put $\gamma_{\Phi}\left(X^{*}, Y\right)=1$ if $X^{*}$ is elliptic and $Y$ is split, and otherwise put $\gamma_{\Phi}\left(X^{*}, Y\right)=0$ if $X^{*}$ and $Y$ do not lie in stably conjugate tori.

Remark 6.6. The dependence of $\gamma_{\Phi}\left(X^{*}, Y\right)$ on $X^{*}$ is via $r^{\prime}$ and $\Phi^{\prime}$ (see Notation 5.2). Expanding these definitions shows that $\gamma_{\Phi}\left(X^{*}, Y\right)=c_{\theta, \phi} \cdot \operatorname{sgn}_{\theta}(\beta s)$ when $X^{*}$ and $Y$ lie in stably conjugate tori, using Notations 5.1 and 5.8.

We have defined $\gamma_{\Phi}\left(X^{*}, Y\right)$ only when $X^{*}$ and $Y$ belong to (possibly different) standard tori, in the sense of Definition 4.1. A direct computation shows that, if we replace $X^{*}$ or $Y$ by a rational conjugate, or replace the pair $\left(X^{*}, Y\right)$ by a stable conjugate, such that $X^{*}$ and $Y$ still lie in standard tori, then the constant $\gamma_{\Phi}\left(X^{*}, Y\right)$ does not change. (In the notation of Definition 8.2, $\operatorname{Ad}^{*}(g) X^{*}$ lies in a standard torus if and only if $\varphi_{\theta}(g)=(\alpha, 0)$, in which case $\operatorname{Ad}^{*}(g) X^{*}=\beta N_{\theta}(\alpha) \cdot \sqrt{N_{\theta}(\alpha)^{-2} \theta}$; and similarly for $Y$.) This allows us to define $\gamma_{\Phi}\left(X^{*}, Y\right)$ for all pairs of regular, semisimple elements, if desired.

By Lemma 6.2,

$$
\gamma_{\mathrm{ram}}(s)^{2}=\operatorname{sgn}_{\varpi}(-1) .
$$

To make use of Propositions 7.5 and 7.7 below, we need the computation

$$
\begin{aligned}
& \operatorname{sgn}_{\varpi}(v) G_{\varpi}\left(\Phi_{\varpi}^{\prime} r^{\prime}+1\right.=\operatorname{sgn}_{\varpi}\left(\varpi^{-\left(r^{\prime}+1\right)} s \theta\right) \cdot \operatorname{sgn}_{\varpi}\left(\varpi^{r^{\prime}+1}\right) G_{\varpi}\left(\Phi^{\prime}\right) \\
&=\operatorname{sgn}_{\varpi}(-\theta) \gamma_{\mathrm{ram}}(s) .
\end{aligned}
$$

Remark 6.9. We will be interested exclusively in the case when $\theta \in\{1, \epsilon, \varpi\}$. This means we seem to be omitting the cases when $\theta \in\left\{\varpi^{2} \epsilon, \epsilon^{2} \varpi, \epsilon^{ \pm 1} \varpi\right\}$, but, actually, this problem is not serious. Indeed, for $b \in k$, write

$$
g_{b}:=\left(\begin{array}{ll}
1 & 0 \\
0 & b
\end{array}\right) \in \mathrm{GL}_{2}(k)
$$

Then

$$
\operatorname{Ad}^{*}\left(g_{b}\right) X^{*}=\operatorname{Ad}^{*}\left(g_{b}\right)(\beta \cdot \sqrt{\theta})=\beta b^{-1} \cdot \sqrt{b^{2} \theta}
$$


(where we identify $\mathfrak{t}_{\theta}^{*}$ with $\mathfrak{t}_{\theta}$ via the trace pairing, as in Notation 5.1); and $\hat{\mu}_{X^{*}}^{G}=$ $\hat{\mu}_{\mathrm{Ad}^{*}\left(g_{b}\right) X^{*}} \circ \operatorname{Ad}\left(g_{b}\right)$. This covers $\theta=\varpi^{2} \epsilon$ (by taking $\left.b=\varpi^{-1}\right)$ and $\theta=\epsilon^{2} \varpi$ (by taking $b=\epsilon^{-1}$ ). Handling $\theta \in\left\{\epsilon^{ \pm 1} \varpi\right\}$ requires a different observation: since our choice of uniformiser was arbitrary, it could as well have been $\epsilon^{ \pm 1} \varpi$ (or, for that matter, $\left.\epsilon^{2} \varpi\right)$ as $\varpi$ itself. Thus, the formulae for the cases $\theta=\epsilon^{n} \varpi$ can be obtained by simple substitution.

The definition of $\gamma_{\Phi}\left(X^{*}, Y\right)$ when $\theta \equiv \epsilon \varpi\left(\bmod \left(k^{\times}\right)^{2}\right)$ is an instance of this; namely, by Lemma 6.2,

$$
\begin{aligned}
-\gamma_{\mathrm{un}}(s) \gamma_{\mathrm{ram}}(s) & =(-1)^{r^{\prime}} \operatorname{sgn}_{\epsilon}(s) \cdot \operatorname{sgn}_{\varpi}(-s) G_{\varpi}\left(\Phi^{\prime}\right) \\
& =\operatorname{sgn}_{\epsilon \varpi}(-s) \cdot \operatorname{sgn}_{\varpi}(\epsilon)^{r^{\prime}} G_{\varpi}\left(\Phi^{\prime}\right)=\operatorname{sgn}_{\epsilon \varpi}(-s) G_{\epsilon \varpi}\left(\Phi^{\prime}\right),
\end{aligned}
$$

where we have used that $\operatorname{sgn}_{\epsilon}(-1)=1$ and $\operatorname{sgn}_{\varpi}(\epsilon)=-1$.

We next define a constant $c_{0}\left(X^{*}\right)$ for use in Theorems 9.7 and 10.10. Those theorems (and Proposition 11.2) show that, as the notation suggests, it is the coefficient of the trivial orbit in the expansion of the germ of $\hat{\mu}_{X^{*}}^{G}$ in terms of Fourier transforms of nilpotent orbital integrals (see [Harish-Chandra 1999, Theorem 5.11]).

\section{Definition 6.10.}

$$
c_{0}\left(X^{*}\right)= \begin{cases}-2 q^{-1} & X^{*} \text { split, } \\ -q^{-1} & X^{*} \text { unramified } \\ -\frac{1}{2} q^{-2}(q+1) & X^{*} \text { ramified. }\end{cases}
$$

Recall that $\hat{\mu}_{X^{*}}^{G}$ is defined in terms of the measure $\mathrm{d} \dot{g}$ of Proposition 11.2; in the notation of that proposition, whenever $X^{*}$ is elliptic,

$$
c_{0}\left(X^{*}\right)=(q-1)^{-1} \operatorname{meas}_{\mathrm{d} \dot{g}}(\dot{K}) .
$$

\section{Bessel functions}

Our strategy for computing Fourier transforms of orbital integrals is to reduce them to $p$-adic Bessel functions (see Proposition 8.11, (9.3), and (10.2)). In this context, we are referring to the complex-valued Bessel functions defined in [Sally and Taibleson 1966, Section 4], not the $p$-adic-valued ones defined in [Dwork 1974].

The definition of these functions depends on an additive character, denoted by $\chi$ in [Sally and Taibleson 1966], and a multiplicative character $\pi$ of $k$. For internal consistency, we will instead denote the additive character by $\Phi$ and the multiplicative character by $\chi$; but, for consistency with their work, we require throughout this section that $\mathrm{d}(\Phi)=-1$, that is, $\Phi$ is trivial on $R$ but not on $\wp^{-1}$. 
Definition 7.1 [Sally and Taibleson 1966, (4.1)]. For $\chi \in \widehat{k^{x}}$, the $p$-adic Bessel function of order $\chi$ is given by

$$
J_{\chi}(u, v)=\oint_{k^{\times}} \Phi\left(u x+v x^{-1}\right) \chi(x) \mathrm{d}^{\times} x \quad \text { for } u, v \in k^{\times},
$$

where $\mathrm{d}^{\times} x$ is the Haar measure on $k^{\times}$fixed in Definition 2.1. Also put $J_{\chi}^{\theta}=$ $\frac{1}{2}\left(J_{\chi}+J_{\chi} \operatorname{sgn}_{\theta}\right)$, with notation as in Definition 3.2.

The locally constant $K$-Bessel function $K(z \mid \chi)$ of [Trimble 1994, Definition 3.2] is $J_{\chi}\left(\varpi^{t}, \varpi^{t}\right)$ (in the notation of that definition), where $\varpi$ is a uniformiser.

For $\chi \neq 1$, it is natural to extend the Bessel function by putting $J_{\chi}(u, 0)=$ $\chi(u)^{-1} \Gamma(\chi)$ and $J_{\chi}(0, v)=\chi(v) \Gamma\left(\chi^{-1}\right)$, where the $\Gamma$-factors are as in [Sally and Taibleson 1966, Section 3]. Under some conditions on $\chi$, we can even define $J_{\chi}(0,0)$ (either as 0 or the sum of a geometric series), but we do not need to do this.

The notation $J_{\chi}^{\theta}$ arises naturally in our computations; see Proposition 8.11.

Definition 7.2. We say that a character $\chi \in \widehat{k^{x}}$ is mildly ramified if $\chi$ is trivial on $1+\wp$, but nontrivial on $k^{\times}$.

Since our orbital-integral calculations require information about $J_{\chi}$ only for $\chi$ mildly ramified, and since more precise information in that case is available in general, we focus our attention there.

Notation 7.3. Fix the following notation for the remainder of the section.

- $u, v \in k^{\times}$,

- $m=-\operatorname{ord}(u v)$, and

- $\chi \in \widehat{k^{x}}$.

This is consistent with Notation 8.6. After Proposition 7.5, we will assume that $\chi$ is mildly ramified.

Of particular interest to us later will be the cases where $\chi$ is an unramified twist of one of the characters $\operatorname{sgn}_{\theta^{\prime}}$ of Definition 3.2 (that is, is of the form $v^{\alpha} \operatorname{sgn}_{\theta}$ for some $\alpha \in \mathbb{C})$. Note that $\operatorname{sgn}_{\epsilon}=v^{\pi i / \ln (q)}$.

Theorem 7.4 [Sally and Taibleson 1966, Theorems 4.8 and 4.9].

$$
J_{\chi}(u, v)= \begin{cases}\chi(v) \Gamma\left(\chi^{-1}\right)+\chi(u)^{-1} \Gamma(\chi) & m \leq 1, \\ \chi(u)^{-1} F_{\chi}(m / 2, u v) & m \geq 2 \text { and } m \text { even, } \\ 0 & m>2 \text { and } m \text { odd },\end{cases}
$$

where the $\Gamma$-factors are as in [Sally and Taibleson 1966, Section 3], and

$$
F_{\chi}(m / 2, u v):=\int_{\operatorname{ord}(x)=-m / 2} \Phi\left(x+u v x^{-1}\right) \chi(x) \mathrm{d}^{\times} x .
$$


The $\Gamma$-factor tables of [Sally and Taibleson 1966, Theorem 3.1], together with Lemma 6.3, mean that we understand $J_{\chi}(u, v)$ completely when $m<2$, but further calculation is necessary in the remaining cases.

\section{Proposition 7.5. If}

- $h \in \mathbb{Z}_{>0}$,

- $\chi$ is trivial on $1+\wp^{h}$, and

- $m \geq 4 h-1$,

then $J_{\chi}(u v)=0$ if $u v \notin\left(k^{\times}\right)^{2}$; and, if $w \in k^{\times}$satisfies $u v=w^{2}$, then

$$
\begin{aligned}
J_{\chi}(u, v)=q^{-m / 4} & \chi\left(u^{-1} w\right) \\
& \times \begin{cases}\Phi(2 w)+\chi(-1) \Phi(-2 w) & 4 \mid m, \\
\operatorname{sgn}_{\varpi}(w) G_{\varpi}(\Phi)\left(\Phi(2 w)+\left(\chi \operatorname{sgn}_{\varpi}\right)(-1) \Phi(-2 w)\right) & 4 \nmid m .\end{cases}
\end{aligned}
$$

Proof. If $m$ is odd, then the vanishing result follows from Theorem 7.4, so we assume that $m$ is even. In this case, $m \geq 4 h$, and, by Theorem 7.4, $J_{\chi}(u, v)=$ $\chi(u)^{-1} F_{\chi}(m / 2, u v)$.

We evaluate the integral defining $F_{\chi}(m / 2, u v)$ by splitting it into pieces. Write

$$
\begin{aligned}
& S_{u v}=\left\{x \in k: \operatorname{ord}(x)=-m / 2 \text { and } \operatorname{ord}\left(x-u v x^{-1}\right)<-m / 2+h\right\}, \\
& T_{u v}=\left\{x \in k: \operatorname{ord}(x)=-m / 2 \text { and } \operatorname{ord}\left(x-u v x^{-1}\right) \geq-m / 2+h\right\} .
\end{aligned}
$$

Both $S_{u v}$ and $T_{u v}$ are invariant under multiplication by $1+\wp$, and if $x \in T_{u v}$, then $u v \in x^{2}\left(1+\wp^{h}\right) \subseteq\left(k^{\times}\right)^{2}$. We claim that the relevant integral may be taken over only $T_{u v}$.

If $X \in \wp^{m / 2-h}$, then by Lemma 2.6 and the fact that $2(m / 2-h) \geq m / 2$, we have

$$
\mathrm{c}(X) \equiv 1+2 X\left(\bmod \wp^{m / 2}\right) \quad \text { and } \quad \mathrm{c}(X)^{-1} \equiv 1-2 X\left(\bmod \wp^{m / 2}\right),
$$

SO

$$
\begin{aligned}
\int_{S_{u v}} \Phi & \left(x+u v x^{-1}\right) \chi(x) \mathrm{d}^{\times} x \\
& =(\star) \int_{\wp^{m / 2-h}} \int_{S_{u v}} \Phi\left(x \cdot \mathrm{c}(X)+u v x^{-1} \cdot \mathrm{c}(X)^{-1}\right) \chi(x \cdot \mathrm{c}(X)) \mathrm{d}^{\times} x \mathrm{~d} X \\
& =(\star) \int_{S_{u v}} \Phi\left(x+u v x^{-1}\right) \chi(x) \int_{\wp^{m / 2-h}} \Phi_{2\left(x-u v x^{-1}\right)}(X) \mathrm{d} X \mathrm{~d}^{\times} x,
\end{aligned}
$$

where $(\star)=\operatorname{meas}_{\mathrm{d} X}\left(\wp^{m / 2-h}\right)^{-1}$ is a constant and we used that $\Phi$ is trivial on $x \wp^{m / 2} \cup u v x^{-1} \wp^{m / 2} \subseteq R$ and $\chi$ is trivial on $\mathrm{c}\left(\wp^{m / 2-h}\right)=1+\wp^{m / 2-h} \subseteq 1+\wp^{h}$. By (2.3), we have that $\mathrm{d}\left(\Phi_{2\left(x-u v x^{-1}\right)}\right)>m / 2-h+1$ (that is, $\Phi_{2\left(x-u v x^{-1}\right)}$ is a nontrivial character on $\wp^{m / 2-h}$ ) whenever $x \in S_{u v}$, so the inner integral is 0 . This shows that, as desired, the integral defining $F_{\chi}(m / 2, u v)$ may be taken over only $T_{u v}$. 
If $u v \notin\left(k^{\times}\right)^{2}$, then $T_{u v}=\varnothing$, so $J_{\chi}(u, v)=\chi(u)^{-1} F_{\chi}(m / 2, u v)=0$; whereas, if $w \in k^{\times}$satisfies $w^{2}=u v$, then $T_{u v}=w\left(1+\wp^{h}\right) \sqcup-w\left(1+\wp^{h}\right)$, so

$$
\begin{aligned}
J_{\chi}(u, v)=\chi(u)^{-1}\left(\int_{w\left(1+\wp^{h}\right)} \Phi(x+\right. & \left.u v x^{-1}\right) \chi(x) \mathrm{d}^{\times} x \\
& \left.+\int_{-w\left(1+\wp^{h}\right)} \Phi\left(x+u v x^{-1}\right) \chi(x) \mathrm{d}^{\times} x\right) .
\end{aligned}
$$

Note that $\operatorname{ord}(w)=-m / 2$.

We show a detailed calculation of the first integral; of course, that of the second is identical. Note that the integral no longer involves $\chi$. By Lemma 2.6 again, $X \mapsto w \cdot \mathrm{c}(X)$ is a measure-preserving bijection from $\wp^{h}$ to $w\left(1+\wp^{h}\right)$, so

$$
\int_{w\left(1+\wp^{h}\right)} \Phi\left(x+u v x^{-1}\right) \chi(x) \mathrm{d}^{\times} x=\chi(w) \int_{\wp^{h}} \Phi_{w}\left(\mathrm{c}(X)+\mathrm{c}(X)^{-1}\right) \mathrm{d} X,
$$

where we used $u v w^{-1}=w$ and again that $\chi$ is trivial on $\mathrm{c}\left(\wp^{h}\right)=1+\wp^{h}$. We will evaluate the latter integral by breaking it into "shells" on which ord $(X)$ is constant, using the following facts. By direct computation (and Definition 2.5),

$$
\mathrm{c}(X)+\mathrm{c}(X)^{-1}=2 \mathrm{c}\left(X^{2}\right)
$$

for $X \in k \backslash\{1\}$. If $\operatorname{ord}(X)=i$ and $\operatorname{ord}(Y)=j$, then Lemma 2.6 implies

$$
\begin{gathered}
\mathrm{c}\left((X+Y)^{2}\right) \equiv \mathrm{c}\left(X^{2}+2 X Y\right)\left(\bmod \wp^{2 j}\right), \\
\mathrm{c}\left(X^{2}+2 X Y\right) \equiv \mathrm{c}\left(X^{2}\right)+4 X Y\left(\bmod \wp^{2 j}\right) .
\end{gathered}
$$

(The second congruence could be made much finer, but we do not need this.)

In particular, fix $i \geq h$ with $2 i<m / 2-1$, so that $\mathrm{d}(\Phi)=m / 2-1<2(m / 2-1-i)$ (that is, $\Phi$ is trivial on $\left.\wp^{2(m / 2-1-i)}\right)$. Then

$$
\begin{aligned}
\int_{\operatorname{ord}(X)=i} \Phi_{w}(\mathrm{c}(X)+ & \left.\mathrm{c}(X)^{-1}\right) \mathrm{d} X \\
& =(\star) \int_{\wp^{m / 2-1-i}} \int_{\operatorname{ord}(X)=i}\left(\Phi_{2 w} \circ \mathrm{c}\right)\left((X+Y)^{2}\right) \mathrm{d} X \mathrm{~d} Y \\
& =(\star) \int_{\operatorname{ord}(X)=i}\left(\Phi_{2 w} \circ \mathrm{c}\right)\left(X^{2}\right) \int_{\wp^{m / 2-1-i}} \Phi_{8 w X}(Y) \mathrm{d} Y \mathrm{~d} X,
\end{aligned}
$$

where $(\star)=\operatorname{meas}\left(\wp^{m / 2-1-i}\right)^{-1}$ is a constant. Since $d\left(\Phi_{8 w X}\right)=\mathrm{d}\left(\Phi_{w}\right)-\operatorname{ord}(8 X)=$ $m / 2-1-i$, the inner integral is 0 .

Note that $\lceil(m / 2-1) / 2\rceil \geq h$. Thus

$$
J_{\chi}(u, v)=\int_{\wp\lceil(m / 2-1) / 2\rceil}\left(\Phi_{2 w} \circ \mathrm{c}\right)\left(X^{2}\right) \mathrm{d} X .
$$


If $m / 2$ is even, then the integral is over $\wp^{m / 4}$, and $\mathrm{c}\left(X^{2}\right) \equiv 1\left(\bmod \wp^{m / 2} \subseteq \operatorname{ker} \Phi_{2 w}\right)$ for all $X \in \wp^{m / 4}$. Thus, in that case,

$$
J_{\chi}(u, v)=\operatorname{meas}_{\mathrm{d} X}\left(\wp^{m / 4}\right) \Phi_{2 w}(1)=q^{-m / 4} \Phi(2 w) .
$$

If $m / 2$ is odd, then the integral is over $\wp^{m / 4-1 / 2}$, and $\mathrm{c}\left(X^{2}\right) \equiv 1+2 X^{2}\left(\bmod \wp^{m / 2}\right)$ for all $X \in \wp^{m / 4-1 / 2}$. So, in that case,

$$
\begin{aligned}
J_{\chi}(u, v) & =\operatorname{meas}_{\mathrm{d} X}\left(\wp^{m / 4+1 / 2}\right) \Phi_{2 w}(1) \sum_{X \in \wp^{m / 4-1 / 2} / \wp^{m / 4+1 / 2}} \Phi_{4 w}\left(X^{2}\right) \\
& =q^{-m / 4} \Phi(2 w) q^{-1 / 2} \sum_{X \in R / \wp} \Phi_{4 w \varpi^{m / 2-1}}\left(X^{2}\right) .
\end{aligned}
$$

By Lemma 6.2, and the fact that $m / 2$ is odd, this can be rewritten as

$$
q^{-m / 4} \Phi(2 w) \operatorname{sgn}_{\varpi}(-1)^{m / 2-1} G_{\varpi}\left(\Phi_{4 w}\right)=q^{-m / 4} \Phi(2 w) \operatorname{sgn}_{\varpi}(w) G_{\varpi}(\Phi) .
$$

The result now follows from $(*)$.

From now on, we assume that $\chi$ is mildly ramified. In particular, we may take $h=1$, so that Proposition 7.5 holds whenever $m>2$.

Definition 7.6. For

- $\xi \in \mathfrak{f}^{\times}$,

- $\bar{\Phi}$ an (additive) character of $\mathfrak{f}$, and

- $\bar{\chi}$ a (multiplicative) character of $\mathfrak{f}^{\times}$,

define the corresponding twisted Kloosterman sum by

$$
K(\bar{\chi}, \bar{\Phi} ; \xi):=\sum_{x \in \mathfrak{f}^{\times}} \bar{\Phi}\left(x+\xi x^{-1}\right) \bar{\chi}(x) .
$$

Proposition 7.7. If $m=2$, then

$$
J_{\chi}(u, v)=q^{-1} \chi(u \varpi)^{-1} K(\bar{\chi}, \bar{\Phi} ; \xi) .
$$

Here,

- $\xi$ is the image in $\mathfrak{f}^{\times}$of $\varpi^{2} u v \in R^{\times}$,

- $\bar{\Phi}$ is the (additive) character of $\mathfrak{f}=R / \wp$ arising from the restriction to $R$ of the depth-0 character $\Phi_{\varpi^{-1}}$ of $k$, and

- $\bar{\chi}$ is the (multiplicative) character of $\mathfrak{f}^{\times} \cong R^{\times} / 1+\wp$ arising from the restriction to $R^{\times}$of $\chi$. 
Proof. By Theorem 7.4,

$$
\begin{aligned}
\chi(u \varpi) J_{\chi}(u, v) & =\chi(\varpi) \int_{\operatorname{ord}(x)=-1} \Phi\left(x+u v x^{-1}\right) \chi(x) \mathrm{d}^{\times} x \\
& =\int_{R^{\times}} \Phi\left(\varpi^{-1} x+u v \cdot \varpi x^{-1}\right) \chi(x) \mathrm{d}^{\times} x \\
& =\operatorname{meas}_{\mathrm{d}^{\times} x}(1+\wp) \sum_{x \in R^{\times} / 1+\wp} \Phi_{\varpi^{-1}}\left(x+\varpi^{2} u v x^{-1}\right) \chi(x) \mathrm{d}^{\times} x .
\end{aligned}
$$

Since $\operatorname{meas}_{\mathrm{d}^{\times} x}(1+\wp)=q^{-1}$, the result follows.

Corollary 7.8. Suppose $m=2$. Then for $\alpha \in \mathbb{C}$,

$$
\begin{gathered}
J_{v^{\alpha}}(u, v)=q^{\alpha-1}|u|^{-\alpha} \sum_{\substack{c \in \wp^{-1} / R \\
c^{2} \neq u v}} \Phi(2 c) \operatorname{sgn}_{\varpi}\left(c^{2}-u v\right), \\
J_{v^{\alpha} \operatorname{sgn}_{\varpi}}(u, v)=q^{\alpha-1 / 2}|u|^{-\alpha} \operatorname{sgn}_{\varpi}(v) G_{\varpi}(\Phi) \sum_{\substack{c \in \wp^{-1} / R \\
c^{2}=u v}} \Phi(2 c) .
\end{gathered}
$$

Proof. If $\chi=v^{\alpha}$, then $\bar{\chi}=1$, so [Lidl and Niederreiter 1997, Theorem 5.47] gives

$$
\begin{aligned}
K(\bar{\chi}, \bar{\Phi} ; \xi) & =\sum_{\substack{c \in \mathfrak{f} \\
c^{2} \neq \xi}} \bar{\Phi}(2 c) \operatorname{sgn}_{\mathfrak{f}}\left(c^{2}-\alpha\right) \\
& =\sum_{\substack{c \in R / \wp \\
c^{2} \neq \varpi^{2} u v}} \bar{\Phi}(2 c) \operatorname{sgn}_{\varpi}\left(c^{2}-\varpi^{2} u v\right) \\
& =\sum_{\substack{c \in \wp^{-1} / R \\
c^{2} \neq u v}} \Phi(2 c) \operatorname{sgn}_{\varpi}\left(c^{2}-u v\right) .
\end{aligned}
$$

(Note that our $\bar{\Phi}$ is their $\chi$, and they write $K(\chi ; a, b)$ where we write $K(\bar{\Phi}, 1 ; a b)$.) If $\chi=v^{\alpha} \operatorname{sgn}_{\varpi}$, then $\bar{\chi}=\operatorname{sgn}_{\mathfrak{f}}$, therefore [Lidl and Niederreiter 1997, Exercises 5.84-85] gives that

$$
K(\bar{\chi}, \bar{\Phi} ; \xi)=\operatorname{sgn}_{\mathfrak{f}}(\xi) G\left(\operatorname{sgn}_{\mathfrak{f}}, \bar{\Phi}\right) \sum_{\substack{c \in \mathfrak{f} \\ c^{2}=\xi}} \bar{\Phi}(2 c)=\operatorname{sgn}_{\varpi}(u v) G\left(\operatorname{sgn}_{\mathfrak{f}}, \bar{\Phi}\right) \sum_{\substack{c \in \wp^{-1} / R \\ c^{2}=u v}} \Phi(2 c),
$$

where $G\left(\operatorname{sgn}_{\mathfrak{f}}, \bar{\Phi}\right)=\sum_{X \in \mathfrak{f}^{x}} \bar{\Phi}(X) \operatorname{sgn}_{\mathfrak{f}}(X)$. (Note that our $\bar{\Phi}$ is their $\chi$ and our $\bar{\chi}$ their $\eta$, and our $K(\bar{\chi}, \bar{\Phi} ; \xi)$ is their $K(\eta, \chi ; 1, \xi)$.) Since $\mathrm{d}(\Phi)=-1$, Lemma 6.2 gives that $G\left(\operatorname{sgn}_{\mathfrak{f}}, \bar{\Phi}\right)=q^{1 / 2} \operatorname{sgn}_{\varpi}(-1) G_{\varpi}(\Phi)$.

The result now follows from Proposition 7.7. 
The following apparently specialised corollary allows simplification of many of our "shallow" computations (see Section 9A and Section 10A).

Corollary 7.9. If $m \geq 2$ and $\operatorname{ord}(u)=\operatorname{ord}(v)$, then $J_{v^{\alpha} \chi}(u, v)$ is independent of $\alpha \in \mathbb{C}$; in particular,

$$
J_{\chi}^{\epsilon}(u, v)=J_{\chi}(u, v) \quad \text { and } \quad J_{\chi}^{\varpi}(u, v)=J_{\chi \operatorname{sgn}_{\epsilon}}^{\varpi}(u, v) .
$$

If $m \geq 2$ and $\operatorname{ord}(u)=\operatorname{ord}(v)+2$, then $J_{v^{\alpha} \chi}(u, v)=q^{\alpha} J_{\chi}(u, v)$; in particular,

$$
J_{\chi}^{\epsilon}(u, v)=0 \quad \text { and } \quad J_{\chi}^{\varpi}(u, v)=-J_{\chi \operatorname{sgn}_{\epsilon}}^{\varpi}(u, v) .
$$

Proof. Suppose that $m>2$. If $u v \notin\left(k^{\times}\right)^{2}$, then $J_{v^{\alpha} \chi}(u, v)=0$ for all $\alpha \in \mathbb{C}$. If $u v=w^{2}$, then the only dependence on $\alpha$ in Proposition 7.5 is via the factor $\chi\left(u^{-1} w\right)$. If $\operatorname{ord}(u)=\operatorname{ord}(v)$, then also ord $(w)=\operatorname{ord}(u)$, so $v^{\alpha}\left(u^{-1} w\right)=1$. If $\operatorname{ord}(u)=\operatorname{ord}(v)+2$, then $\operatorname{ord}(w)=\operatorname{ord}(u)-1$, so $v^{\alpha}\left(u^{-1} w\right)=q^{\alpha}$.

Now suppose that $m=2$, that is, that $\operatorname{ord}(u v)=-2$. Since $\overline{v^{\alpha} \chi}=\bar{\chi}$, the only dependence on $\alpha$ in Proposition 7.7 is via the factor $\chi(u \varpi)^{-1}$. If $\operatorname{ord}(u)=\operatorname{ord}(v)$, then $\operatorname{ord}(u)=-1$, so $v^{\alpha}(u \varpi)=1$. If $\operatorname{ord}(u)=\operatorname{ord}(v)+2$, then $\operatorname{ord}(u)=0$, so $v^{\alpha}(u \varpi)=q^{-\alpha}$.

\section{A mock Fourier transform}

We introduce a function $M_{X^{*}}^{G}$ specified by an integral formula (see Definition 8.4) reminiscent of the usual one for (the function representing) $\hat{\mu}_{X^{*}}^{G}$ (see [Adler and DeBacker 2004, Theorem A.1.2]). We will eventually show (see Proposition 11.2) that it is actually equal to $\hat{\mu}_{X^{*}}^{G}$, but first we spend some time computing it.

In the notation of Definition 4.1, we have

$$
\operatorname{tr} g \cdot \sqrt{\theta} \cdot g^{-1} \cdot \sqrt{\theta^{\prime}}=N_{\theta}(\alpha) \cdot \theta^{\prime}+N_{\theta}(\gamma),
$$

where

$$
g=\left(\begin{array}{ll}
a & b \\
c & d
\end{array}\right)
$$

$\alpha=a+b \sqrt{\theta}$, and $\gamma=c+d \sqrt{\theta}$. Since $1=a d-b c=\operatorname{Im}_{\theta}(\bar{\alpha} \cdot \gamma)$, we have that $\gamma=\bar{\alpha}^{-1} \cdot(t+\sqrt{\theta})$ for some $t \in k$; specifically, $t=\operatorname{Re}_{\theta}(\bar{\alpha} \cdot \gamma)=a c-b d \theta$. This calculation motivates the definition of the following map.

Definition 8.2. Define $\varphi_{\theta}: G \rightarrow k_{\theta}^{\times} \times k$ by

$$
\varphi_{\theta}(g)=(a+b \sqrt{\theta}, a c-b d \theta)
$$

for

$$
g=\left(\begin{array}{ll}
a & b \\
c & d
\end{array}\right) \in G
$$


Note that $\varphi_{\theta}$ is a bianalytic map (of $k$-manifolds), with inverse

$$
(\alpha, t) \mapsto\left(\begin{array}{c}
\operatorname{Re}_{\theta}(\alpha) \\
N_{\theta}(\alpha)^{-1}\left(t \cdot \operatorname{Re}_{\theta}(\alpha)+\theta \cdot \operatorname{Im}_{\theta}(\alpha)\right) \\
N_{\theta}(\alpha)^{-1}\left(\operatorname{Re}_{\theta}(\alpha)+t \cdot \operatorname{Im}_{\theta}(\alpha)\right)
\end{array}\right) .
$$

It is not an isomorphism, but its restrictions to $T_{\theta}, A$, and

$$
\left\{\left(\begin{array}{ll}
1 & 0 \\
b & 1
\end{array}\right): b \in k\right\}
$$

are isomorphisms onto $C_{\theta} \times\{0\}, k^{\times} \times\{0\}$, and $\{1\} \times k$, respectively. In fact, the next lemma says a bit more.

Lemma 8.3. If $g \in G$ satisfies $\varphi_{\theta}(g)=(\alpha, t)$, and

- $h \in T_{\theta}$ is identified with $\eta \in C_{\theta}$,

- $a=\left(\begin{array}{ll}\lambda & 0 \\ 0 & \lambda\end{array}\right)$ with $\lambda \in k^{\times}$, and

- $\bar{u}=\left(\begin{array}{ll}1 & 0 \\ b & 1\end{array}\right)$ with $b \in k$,

then

$$
\begin{aligned}
& \varphi_{\theta}(g h)=(\alpha \eta, t), \\
& \varphi_{\theta}(a g)=(\lambda \alpha, t), \\
& \varphi_{\theta}(\bar{u} g)=\left(\alpha, t+N_{\theta}(\alpha) b\right) .
\end{aligned}
$$

Proof. This is a straightforward computation.

We can now define our "mock orbital integral". Again, Proposition 11.2 will eventually show that it is actually equal to the function in which we are interested.

Definition 8.4. For $\alpha \in k_{\theta}^{\times}$and $t \in k$, put

$$
\left\langle X^{*}, Y\right\rangle_{\alpha, t}:=\beta s\left(N_{\theta}(\alpha) \cdot \theta^{\prime}+N_{\theta}(\alpha)^{-1} \cdot \theta-N_{\theta}(\alpha)^{-1} \cdot t^{2}\right) .
$$

The dependence on $\alpha$ is only via $N_{\theta}(\alpha)$. Thus, we may define

$$
M_{X^{*}}^{G}(Y):=\oint_{k_{\theta}^{\times} / C_{\theta}} \oint_{k} \Phi\left(\left\langle X^{*}, Y\right\rangle_{\alpha, t}\right) \mathrm{d} t \mathrm{~d}^{\times} \dot{\alpha},
$$

where

$$
\begin{aligned}
\oint_{k} f(x) \mathrm{d} t & :=\sum_{n \in \mathbb{Z}} \int_{\operatorname{ord}(x)=n} f(x) \mathrm{d} t, \\
\oint_{k^{\times}} f(x) \mathrm{d}^{\times} x & :=\sum_{n \in \mathbb{Z}} \int_{\operatorname{ord}(x)=n} f(x) \mathrm{d}^{\times} x, \\
\oint_{k_{\theta}^{\times} / C_{\theta}}\left(f \circ N_{\theta}\right)(\alpha) \mathrm{d}^{\times} \dot{\alpha} & :=\oint_{k^{\times}}\left[N_{\theta}\left(k^{\times}\right)\right](x) f(x) \mathrm{d}^{\times} x
\end{aligned}
$$


(for those $f \in C^{\infty}(k)$ for which the sum converges) are "principal-value" integrals, as in [Sally and Taibleson 1966, p. 282]. Here, $\mathrm{d} t$ and $\mathrm{d}^{\times} x$ are the measures of Definition 2.1, and $[S]$ denotes the characteristic function of $S$.

By (8.1) (and Notations 5.1 and 5.8), we have that

$$
\left\langle X^{*}, Y\right\rangle_{\alpha, t}=\left\langle\operatorname{Ad}^{*}(g) X^{*}, Y\right\rangle \quad \text { when } \varphi_{\theta}(g)=(\alpha, t),
$$

where the pairing $\langle\cdot, \cdot\rangle$ on the right is the usual pairing between $\mathfrak{g}^{*}$ and $\mathfrak{g}$.

Notation 8.6. $u=\varpi^{-\left(r^{\prime}+1\right)} s \theta^{\prime}, v=\varpi^{-\left(r^{\prime}+1\right)} s \theta$, and $m=-\operatorname{ord}(u v)$.

This is a special case of Notation 7.3. These particular values of $u$ and $v$ will be fixed for the remainder of the paper. It follows that

$$
u v=\left(\varpi^{-\left(r^{\prime}+1\right)} s\right)^{2} \cdot \theta \theta^{\prime},
$$

therefore

$$
u v \in\left(k^{\times}\right)^{2} \Leftrightarrow \theta \theta^{\prime} \in\left(k^{\times}\right)^{2} .
$$

We use Lemma 5.9 to compute

$$
\begin{aligned}
\operatorname{ord}(u) & =-\left(r^{\prime}+1\right)+\operatorname{ord}\left(s \theta^{\prime}\right)=-\left(r^{\prime}+1+\frac{1}{2} \operatorname{ord}\left(\theta^{\prime}\right)\right)+\mathrm{d}(Y), \\
m & =2\left(r^{\prime}+1\right)-\operatorname{ord}\left(s^{2} \theta^{\prime}\right)-\operatorname{ord}(\theta)=2\left(r^{\prime}+1-\mathrm{d}(Y)\right)-\operatorname{ord}(\theta) .
\end{aligned}
$$

8A. Mock Fourier transforms and Bessel functions. We can now evaluate the integral occurring in Definition 8.4 in terms of Bessel functions - or, rather, the sums $J_{\chi}^{\theta}$ of Definition 7.1.

Proposition 8.11. Let $J_{\chi}^{\theta}$ be as in Definition 7.1 and $\gamma_{\mathrm{un}}(s)$ and $\gamma_{\mathrm{ram}}(s)$ be as in Definition 6.5. Then

$$
\begin{aligned}
M_{X^{*}}^{G}(Y)=\frac{1}{2}|s|^{-1 / 2} q^{-\left(r^{\prime}+1\right) / 2} & \\
& \times\left(\left(J_{v^{1 / 2}}^{\theta}(u, v)+\gamma_{\mathrm{un}}(s) J_{v^{1 / 2} \operatorname{sgn}_{\epsilon}}^{\theta}(u, v)\right)\right. \\
& \left.\quad+\gamma_{\mathrm{ram}}(s)\left(J_{v^{1 / 2} \operatorname{sgn}_{\sigma}}^{\theta}(u, v)-\gamma_{\mathrm{un}}(s) J_{v^{1 / 2} \operatorname{sgn}_{\epsilon}}^{\theta}(u, v)\right)\right),
\end{aligned}
$$

Proof. Recall the notation $\Phi^{\prime}=\Phi_{\beta}$ from Notation 5.2. By Definition 8.4,

(*) $M_{X^{*}}^{G}(Y)=\oint_{k_{\theta}^{\times} / C_{\theta}} \Phi_{s}^{\prime}\left(N_{\theta}(\alpha) \cdot \theta^{\prime}+N_{\theta}(\alpha)^{-1} \cdot \theta\right) \cdot \oint_{k} \Phi^{\prime}\left(-s N_{\theta}(\alpha)^{-1} t^{2}\right) \mathrm{d} t \mathrm{~d}^{\times} \dot{\alpha}$

$$
=q^{-\left(r^{\prime}+1\right) / 2} \oint_{k^{\times}}\left[N_{\theta}\left(k_{\theta}^{\times}\right)\right](x) j\left(\theta^{\prime}, \theta ; x\right) \mathscr{H}\left(\Phi^{\prime},-s x^{-1}\right) \mathrm{d}^{\times} x,
$$

where

- $j\left(\theta^{\prime}, \theta ; x\right):=\Phi_{s}^{\prime}\left(\theta^{\prime} x+\theta x^{-1}\right)=\Phi\left(\beta s\left(\theta^{\prime} x+\theta x^{-1}\right)\right)$ for $x \in k^{\times}$, and

- $\mathscr{H}\left(\Phi^{\prime}, b\right)=\oint_{k} \Phi^{\prime}\left(b t^{2}\right) \mathrm{d}_{\Phi^{\prime}} t$ for $b \in k^{\times}$is as in [Shalika 2004, p. 6]. 
In particular, $\mathrm{d}_{\Phi^{\prime}} t$ is the $\Phi^{\prime}$-self-dual Haar measure on $k$; by [Shalika 2004, p. 5], it satisfies $\mathrm{d} t=q^{-\left(r^{\prime}+1\right) / 2} \mathrm{~d}_{\Phi^{\prime}} t$. This is the reason for the appearance of $q^{-\left(r^{\prime}+1\right) / 2}$ on the last line of the computation.

The significance of $j$ is that integrating it against a (multiplicative) character $\chi$ of $k^{\times}$corresponds to evaluating a Bessel function of order $\chi$, in the sense of Definition 7.1. To be precise, our character $\Phi^{\prime}$ has depth $r^{\prime}$, not -1 , so we must work instead with $\Phi_{\varpi^{r^{\prime}+1}}^{\prime}$. Then

$$
j\left(\theta^{\prime}, \theta ; x\right)=\Phi_{\varpi r^{r^{\prime}+1}}^{\prime}\left(\left(\varpi^{-\left(r^{\prime}+1\right)} s \theta^{\prime}\right) x+\left(\varpi^{-\left(r^{\prime}+1\right)} s \theta\right) x^{-1}\right)=\Phi_{\varpi^{r^{\prime}+1}}^{\prime}\left(u x+v x^{-1}\right),
$$

where $(u, v)$ is as in Notation 8.6 , so

$$
\oint_{k^{\times}} j\left(\theta^{\prime}, \theta ; x\right) \chi(x) \mathrm{d}^{\times} x=J_{\chi}(u, v)
$$

for $\chi \in \widehat{k^{x}}$.

Now $\frac{1}{2}\left(1+\operatorname{sgn}_{\theta}\right)$ is the characteristic function of $N_{\theta}\left(k_{\theta}^{\times}\right)$, so we may rewrite $(*)$ :

$$
q^{-\left(r^{\prime}+1\right) / 2} \oint_{k^{\times}} \frac{1}{2}\left(1+\operatorname{sgn}_{\theta}(x)\right) \cdot j\left(\theta^{\prime}, \theta ; x\right) \mathscr{H}\left(\Phi^{\prime},-s x^{-1}\right) \mathrm{d}^{\times} x .
$$

By [Shalika 2004, Lemma 1.3.2] and Lemma 6.2, we have

$$
\mathscr{H}\left(\Phi^{\prime}, b\right)=|b|^{-1 / 2} \begin{cases}\operatorname{sgn}_{\varpi}(b) G_{\varpi}\left(\Phi^{\prime}\right) & r^{\prime}-\operatorname{ord}(b) \text { even }, \\ 1 & r^{\prime}-\operatorname{ord}(b) \text { odd } .\end{cases}
$$

We find it useful to describe $\mathscr{H}\left(\Phi^{\prime}, b\right)$ without explicit use of cases. As above, $\frac{1}{2}\left(1+(-1)^{n} \operatorname{sgn}_{\epsilon}\right)$ is the characteristic function of $\left\{b \in k^{\times}: \operatorname{ord}(b) \equiv n(\bmod 2)\right\}$, so we may rewrite

$$
\mathscr{H}\left(\Phi^{\prime}, b\right)=\frac{1}{2}\left(1+(-1)^{r^{\prime}} \operatorname{sgn}_{\epsilon}(b)\right) \operatorname{sgn}_{\varpi}(b) G_{\varpi}\left(\Phi^{\prime}\right)+\frac{1}{2}\left(1-(-1)^{r^{\prime}} \operatorname{sgn}_{\epsilon}(b)\right) .
$$

Plugging this into $(* *)$ with $b=-s t^{-1}$ gives

$$
\begin{aligned}
& M_{X^{*}}^{G}(Y)=\frac{1}{2}|s|^{-1 / 2} q^{-\left(r^{\prime}+1\right) / 2} \\
& \times \oint_{k^{\times}} \frac{1}{2}\left(1+\operatorname{sgn}_{\theta}(x)\right) \\
& \quad \times\left(\left(1-\gamma_{\mathrm{un}}(s) \operatorname{sgn}_{\epsilon}(x)\right) \gamma_{\mathrm{ram}}(s) \operatorname{sgn}_{\varpi}(x)\right.\left.+\left(1+\gamma_{\mathrm{un}}(s) \operatorname{sgn}_{\epsilon}(x)\right)\right) \\
& \times|x|^{1 / 2} j\left(\theta^{\prime}, \theta ; x\right) \mathrm{d}^{\times} x .
\end{aligned}
$$

Expanding the product and applying $(\dagger)$ gives the desired formula.

8B. "Deep" Bessel functions. By Proposition 8.11, one approach to computing $M_{X^{*}}^{G}(Y)$ (hence $\hat{\mu}_{X^{*}}^{G}(Y)$, by Proposition 11.2) is to evaluate many Bessel functions, and this is exactly what we do. As Theorem 7.4 makes clear, the behaviour of Bessel functions is more predictable when $m<2$ than otherwise. We introduce a 
convenient shorthand for referring to Bessel functions in this range; we will only use it in this section, and Sections 9B and 10C.

Notation 8.12. Define

$$
[A ; B]_{\theta, r^{\prime}}\left(\theta^{\prime}\right):=|\theta|^{1 / 2} A+q^{-\left(r^{\prime}+1\right)}\left|D_{\mathfrak{g}}(Y)\right|^{-1 / 2} B\left(\theta^{\prime}\right) .
$$

We usually suppress the subscript on $[A ; B]$ and sometimes write

$$
[A ; B(1), B(\epsilon), B(\varpi), B(\epsilon \varpi)]\left(\theta^{\prime}\right)
$$

for the same quantity.

Proposition 8.13. With Notations 5.2, 5.8, 8.6, and the notation of Definition 6.5, if $m<2$, then

$$
\begin{aligned}
|s|^{-1 / 2} q^{-\left(r^{\prime}+1\right) / 2} J_{v^{1 / 2} \chi}(u, v) & \\
& = \begin{cases}{\left[Q_{3}\left(q^{-1 / 2}\right) ; 1\right]\left(\theta^{\prime}\right)} & \chi=1, \\
\gamma_{\mathrm{un}}(s)\left[\operatorname{sgn}_{\epsilon}(\theta) Q_{3}\left(-q^{-1 / 2}\right) ; \operatorname{sgn}_{\epsilon}\right]\left(\theta^{\prime}\right) & \chi=\operatorname{sgn}_{\epsilon}, \\
\gamma_{\mathrm{ram}}(s)^{-1}\left[\operatorname{sgn}_{\varpi}(\theta) q^{-1} ; \operatorname{sgn}_{\varpi}\right]\left(\theta^{\prime}\right) & \chi=\operatorname{sgn}_{\varpi}, \\
-\gamma_{\mathrm{un}}(s) \gamma_{\mathrm{ram}}(s)^{-1}\left[\operatorname{sgn}_{\epsilon \varpi}(\theta) q^{-1} ; \operatorname{sgn}_{\epsilon \varpi}\right]\left(\theta^{\prime}\right) & \chi=\operatorname{sgn}_{\epsilon \varpi},\end{cases}
\end{aligned}
$$

where

$$
Q_{3}(T)=-T\left(T^{2}+T+1\right) .
$$

The unexpected factor $|s|^{-1 / 2} q^{-\left(r^{\prime}+1\right) / 2}$ on the left-hand side crops up repeatedly in calculations (see, for example, Proposition 8.11), so it simplifies matters to include it in this calculation.

Proof. By Theorem 7.4 and Lemma 5.9,

$$
\begin{aligned}
J_{v^{1 / 2} \chi}(u, v)= & \left(v^{1 / 2} \chi\right)(v) \Gamma\left(v^{-1 / 2} \chi\right)+\left(v^{-1 / 2} \chi\right)(u) \Gamma\left(v^{1 / 2} \chi\right) \\
= & \left(v^{1 / 2} \chi\right)\left(v \theta^{-1}\right) \\
& \quad \times\left(\left(v^{1 / 2} \chi\right)(\theta) \Gamma\left(v^{-1 / 2} \chi\right)+\left(v^{-1 / 2} \chi\right)\left(u v \theta^{-1}\right) \Gamma\left(v^{1 / 2} \chi\right)\right) \\
= & |s|^{1 / 2} q^{\left(r^{\prime}+1\right) / 2} \chi\left(\varpi^{r^{\prime}+1} s\right)\left[\chi(\theta) \Gamma\left(v^{-1 / 2} \chi\right) ; \Gamma\left(v^{1 / 2} \chi\right) \cdot \chi\right]\left(\theta^{\prime}\right)
\end{aligned}
$$

whenever $\chi^{2}=1$.

In particular, using [Sally and Taibleson 1966, Theorem 3.1(i, ii)] to compute the $\Gamma$-factors, we see that $|s|^{-1 / 2} q^{-\left(r^{\prime}+1\right) / 2} J_{v^{1 / 2} \chi}(u, v)$ is given by

$$
\begin{cases}{\left[Q_{3}\left(q^{-1 / 2}\right) ; 1\right]\left(\theta^{\prime}\right)} & \chi=1, \\ \gamma_{\mathrm{un}}(s)\left[\operatorname{sgn}_{\epsilon}(\theta) Q_{3}\left(-q^{-1 / 2}\right) ; \operatorname{sgn}_{\epsilon}\right]\left(\theta^{\prime}\right) & \chi=\operatorname{sgn}_{\epsilon}, \\ \operatorname{sgn}_{\varpi}\left(\varpi^{r^{\prime}+1} s\right) \Gamma\left(v^{1 / 2} \operatorname{sgn}_{\varpi}\right)\left[\operatorname{sgn}_{\varpi}(\theta) q^{-1} ; \operatorname{sgn}_{\varpi}\right]\left(\theta^{\prime}\right) & \chi=\operatorname{sgn}_{\varpi}, \\ \gamma_{\mathrm{un}}(s) \operatorname{sgn}_{\varpi}\left(\varpi^{r^{\prime}+1} s\right) \Gamma\left(v^{1 / 2} \operatorname{sgn}_{\epsilon \varpi}\right)\left[\operatorname{sgn}_{\epsilon \varpi}(\theta) q^{-1} ; \operatorname{sgn}_{\epsilon \varpi}\right]\left(\theta^{\prime}\right) & \chi=\operatorname{sgn}_{\epsilon \varpi} .\end{cases}
$$


By [ibid., Theorem 3.1(ii)] again and the fact that $\operatorname{sgn}_{\epsilon \varpi}=v^{i \pi / \ln (q)} \operatorname{sgn}_{\pi}$, we have $\Gamma\left(v^{1 / 2} \operatorname{sgn}_{\epsilon \varpi}\right)=-\Gamma\left(v^{1 / 2} \operatorname{sgn}_{\varpi}\right)$; and, by Lemma 6.3, Definition 6.5, and (6.7),

$$
\begin{aligned}
\operatorname{sgn}_{\varpi}\left(\varpi^{r^{\prime}+1} s\right) \Gamma\left(v^{1 / 2} \operatorname{sgn}_{\varpi}\right) & =\operatorname{sgn}_{\varpi}(-1)^{r^{\prime}+1} \operatorname{sgn}_{\varpi}(s) \cdot \operatorname{sgn}_{\varpi}(-1)^{r^{\prime}+1} G_{\varpi}\left(\Phi^{\prime}\right) \\
& =\operatorname{sgn}_{\varpi}(s) G_{\varpi}\left(\Phi^{\prime}\right) \\
& =\gamma_{\mathrm{ram}}(s)^{-1} .
\end{aligned}
$$

This shows that $(*)$ reduces to the table in the statement.

\section{Split and unramified orbital integrals}

Throughout this section, we have

$$
\theta=1 \text { or } \theta=\epsilon, \text { so that } r^{\prime}=r .
$$

In the split case, $J_{\chi}^{1}=J_{\chi}$ for $\chi \in \widehat{k^{\times}}$, so Proposition 8.11 gives

$$
\begin{aligned}
& M_{X^{*}}^{G}(Y)=\frac{1}{2}|s|^{-1 / 2} q^{-(r+1) / 2} \\
& \quad \times\left(\left(J_{v^{1 / 2}}(u, v)+\gamma_{\mathrm{un}}(s) J_{v^{1 / 2} \operatorname{sgn}_{\epsilon}}(u, v)\right)\right. \\
& \left.\quad+\gamma_{\mathrm{ram}}(s)\left(J_{v^{1 / 2} \operatorname{sgn}_{\varpi}}(u, v)-\gamma_{\mathrm{un}}(s) J_{v^{1 / 2} \operatorname{sgn}_{\epsilon \varpi}}(u, v)\right)\right) .
\end{aligned}
$$

In the unramified case, $J_{\chi}^{\epsilon}=J_{\chi}^{\epsilon} \operatorname{sgn}_{\epsilon}$ for $\chi \in \widehat{k^{\times}}$, so Proposition 8.11 gives

$$
\begin{aligned}
M_{X^{*}}^{G}(Y)= & \frac{1}{2}|s|^{-1 / 2} q^{-(r+1) / 2} \\
& \times\left(\left(1+\gamma_{\mathrm{un}}(s)\right) J_{v^{1 / 2}}^{\epsilon}(u, v)+\gamma_{\mathrm{ram}}(s)\left(1-\gamma_{\mathrm{un}}(s)\right) J_{v^{1 / 2} \operatorname{sgn}_{\varpi}}^{\epsilon}(u, v)\right) .
\end{aligned}
$$

By (6.8) and (6.7),

$$
\operatorname{sgn}_{\varpi}(v) G_{\varpi}\left(\Phi_{\varpi^{r+1}}^{\prime}\right)= \begin{cases}\operatorname{sgn}_{\varpi}(-1) \gamma_{\mathrm{ram}}(s)=\gamma_{\mathrm{ram}}(s)^{-1} & \theta=1, \\ \operatorname{sgn}_{\varpi}(-\epsilon) \gamma_{\mathrm{ram}}(s)=-\gamma_{\mathrm{ram}}(s)^{-1} & \theta=\epsilon .\end{cases}
$$

9A. Far from zero. The results of this section are special cases for split and unramified orbital integrals of results of Waldspurger [1995, Proposition VIII.1]. We prove analogues of these results for ramified orbital integrals in Section 10A.

The qualitative behaviour of unramified orbital integrals does not change as we pass from elements of depth less than $r$ to those of depth exactly $r$; this is unlike the situation for ramified orbital integrals. See Section 10B.

Theorem 9.5. If $\mathrm{d}\left(X^{*}\right)+\mathrm{d}(Y) \leq 0$ and $X^{*}$ is split or unramified, then $M_{X^{*}}^{G}(Y)=0$ unless $X^{*}$ and $Y$ lie in $G$-conjugate tori.

Proof. Recall that $\theta=1$ if $X^{*}$ is split, and $\theta=\epsilon$ if $X^{*}$ is unramified.

By (8.10), $m \geq 2$; in fact, $m>2$ (indeed, $m$ is odd) unless $\operatorname{ord}\left(\theta^{\prime}\right)$ is even. 
If $m>2$, then Proposition 7.5 and (8.8) show that $M_{X^{*}}^{G}(Y)=0$ unless $\theta \theta^{\prime} \in\left(k^{\times}\right)^{2}$. By Section 4, it therefore suffices to consider the cases when $\theta=\epsilon$ and $\theta^{\prime}=\varpi^{2} \epsilon$, that is, $X^{*}$ and $Y$ lie in stably, but not rationally, conjugate tori; and when $m=2$ and $\left\{\theta, \theta^{\prime}\right\}=\{1, \epsilon\}$, that is, one of $X^{*}$ or $Y$ is split, and the other unramified.

Suppose first that $\theta=\epsilon$ and $\theta^{\prime}=\varpi^{2} \epsilon$, so that $\operatorname{ord}(u)=\operatorname{ord}(v)+2$. By Corollary 7.9, (9.3) becomes $M_{X^{*}}^{G}(Y)=0$.

Now suppose $\left\{\theta, \theta^{\prime}\right\}=\{1, \epsilon\}$ and $m=2$. By Corollary 7.9, $\operatorname{since} \operatorname{ord}(u)=\operatorname{ord}(v)$,

$$
J_{v^{1 / 2}}(u, v)=J_{v^{1 / 2} \operatorname{sgn}_{\epsilon}}(u, v) \quad \text { and } \quad J_{v^{1 / 2} \operatorname{sgn}_{\sigma}}(u, v)=J_{v^{1 / 2} \operatorname{sgn}_{\epsilon \sigma}}(u, v),
$$

so (9.2) agrees with (9.3). We shall work with (9.3), since it is simpler.

By Corollary 7.8 and (8.8), $J_{v^{\alpha} \operatorname{sgn}_{\pi}}(u, v)=0$ for all $\alpha \in \mathbb{C}$, in particular, for $\alpha=1 / 2$ and $\alpha=1 / 2+i \pi / \ln (q)$. By (8.10), $\operatorname{ord}(s)=r$, so, by Definition 6.5, $\gamma_{\mathrm{un}}(s)=-1$, and (9.3) (hence also (9.2)) becomes

$$
M_{X^{*}}^{G}(Y)=\frac{1}{2}|s|^{-1 / 2} J_{v^{1 / 2} \operatorname{sgn}_{\varpi}}^{\epsilon}(u, v)=0 .
$$

Theorem 9.6. If $\mathrm{d}\left(X^{*}\right)+\mathrm{d}(Y) \leq 0$ and $X^{*}$ and $Y$ lie in a common split or unramified torus $\boldsymbol{T}$ (with $T=\boldsymbol{T}(k)$ ), then

$$
M_{X^{*}}^{G}(Y)=q^{-(r+1)}\left|D_{\mathfrak{g}}(Y)\right|^{-1 / 2} \gamma_{\Phi}\left(X^{*}, Y\right) \sum_{\sigma \in W(G, T)} \Phi\left(\left\langle\operatorname{Ad}^{*}(\sigma) X^{*}, Y\right\rangle\right),
$$

where $\gamma_{\Phi}\left(X^{*}, Y\right)$ is as in Definition 6.5.

Proof. The hypothesis implies that $\theta=\theta^{\prime}$, so $u=v$. By Corollary 7.9,

$$
J_{v^{1 / 2}}(u, v)=J_{v^{1 / 2} \operatorname{sgn}_{\epsilon}}(u, v) \quad \text { and } \quad J_{v^{1 / 2} \operatorname{sgn}_{\sigma}}(u, v)=J_{v^{1 / 2} \operatorname{sgn}_{\epsilon \sigma}}(u, v),
$$

so (9.2) agrees with (9.3). We again work with (9.3), since it is simpler.

By Remark 4.7, $W\left(G, T_{\theta}\right)=\left\{1, \sigma_{\theta}\right\}$, where $\operatorname{Ad}^{*}\left(\sigma_{\theta}\right) X^{*}=-X^{*}$.

We may take the square root $w$ of $u v$ in Proposition 7.5 to be just $u$. By (8.10),

$$
q^{-m / 4}=q^{-(r+1) / 2} q^{\operatorname{ord}(s) / 2}=q^{-(r+1) / 2}|s|^{-1 / 2} .
$$

By Notations 5.2 and 8.6,

$$
\Phi_{\varpi^{r+1}}^{\prime}( \pm 2 w)=\Phi^{\prime}( \pm 2 s \theta)=\Phi( \pm 2 \beta s \theta)=\Phi\left( \pm\left\langle X^{*}, Y\right\rangle\right)
$$

(the last equality following, for example, from (8.5)).

Suppose ord $(s) \not \equiv r(\bmod 2)$, so $\gamma_{\text {un }}(s)=1$ and $\gamma_{\Phi}\left(X^{*}, Y\right)=1$. By Corollary 7.9, since $u=v,(9.3)$ (hence also (9.2)) becomes

$$
\begin{aligned}
M_{X^{*}}^{G}(Y) & =\frac{1}{2}|s|^{-1 / 2} q^{-(r+1) / 2} \cdot 2 \cdot J_{v^{1 / 2}}^{\epsilon}(u, v) \\
& =|s|^{-1 / 2} q^{-(r+1) / 2} J_{v^{1 / 2}}(u, v) .
\end{aligned}
$$


Since $m>2$ and $4 \mid m$ by (8.10), combining Proposition 7.5, (*), and (**) gives $(\dagger \dagger)$

$$
\begin{aligned}
J_{v^{1 / 2}}(u, v) & =q^{-(r+1) / 2}|s|^{-1 / 2}\left(\Phi\left(\left\langle X^{*}, Y\right\rangle\right)+\Phi\left(-\left\langle X^{*}, Y\right\rangle\right)\right) \\
& =q^{-(r+1) / 2}|s|^{-1 / 2} \sum_{\sigma \in W\left(G, T_{\theta}\right)} \Phi\left(\left\langle\operatorname{Ad}^{*}(\sigma) X^{*}, Y\right\rangle\right) \\
& =q^{-(r+1) / 2}\left|s \theta^{\prime}\right|^{-1 / 2} \gamma_{\Phi}\left(X^{*}, Y\right) \sum_{\sigma \in W\left(G, T_{\theta}\right)} \Phi\left(\left\langle\operatorname{Ad}^{*}(\sigma) X^{*}, Y\right\rangle\right) .
\end{aligned}
$$

The result (in this case) now follows from Lemma 5.9 by combining $(\dagger)$ and $(\dagger \dagger)$.

Suppose now that $\operatorname{ord}(s) \equiv r(\bmod 2)$, so that $\gamma_{\text {un }}(s)=-1$ and

$$
\gamma_{\Phi}\left(X^{*}, Y\right)= \begin{cases}1 & \theta=1, \\ -1 & \theta=\epsilon .\end{cases}
$$

Again by Corollary 7.9, since $u=v,(9.3)$ (hence also (9.2)) becomes (as in ( $\dagger$ ))

$$
M_{X^{*}}^{G}(Y)=|s|^{-1 / 2} q^{-(r+1) / 2} \gamma_{\mathrm{ram}}(s) J_{v^{1 / 2} \operatorname{sgn}_{\varpi}}(u, v) .
$$

Since $4 \nmid m$ by (8.10), if $m>2$, then combining Proposition 7.5, (*), (9.4), and (**) gives (as in $(\dagger \dagger))$

$$
\begin{aligned}
\left(\dagger \dagger^{\prime}<r\right) & J_{v^{1 / 2} \operatorname{sgn}_{\sigma}}(u, v) \\
= & q^{-(r+1) / 2}\left|s \theta^{\prime}\right|^{-1 / 2} \gamma_{\mathrm{ram}}(s)^{-1} \gamma_{\Phi}\left(X^{*}, Y\right) \sum_{\sigma \in W\left(G, T_{\theta}\right)} \Phi\left(\left\langle\operatorname{Ad}^{*}(\sigma) X^{*}, Y\right\rangle\right) .
\end{aligned}
$$

If $m=2$, then $|s|=q^{-r}$ and $\operatorname{ord}(u)=-1$ by Lemma 5.9, (8.9), and (8.10). Thus, combining Corollary $7.8,(9.4)$, and $(* *)$ gives

$$
\begin{aligned}
\left(\dagger \dagger^{\prime}=r\right) & J_{v^{1 / 2} \operatorname{sgn}_{\varpi}}(u, v) \\
& =q^{-1 / 2} \gamma_{\mathrm{ram}}(s)^{-1} \gamma_{\Phi}\left(X^{*}, Y\right) \sum_{\sigma \in W\left(G, T_{\theta}\right)} \Phi\left(\left\langle\operatorname{Ad}^{*}(\sigma) X^{*}, Y\right\rangle\right) \\
& =q^{-(r+1) / 2}\left|s \theta^{\prime}\right|^{-1 / 2} \gamma_{\mathrm{ram}}(s)^{-1} \gamma_{\Phi}\left(X^{*}, Y\right) \sum_{\sigma \in W\left(G, T_{\theta}\right)} \Phi\left(\left\langle\operatorname{Ad}^{*}(\sigma) X^{*}, Y\right\rangle\right) .
\end{aligned}
$$

The result follows by combining $\left(\dagger^{\prime}\right)$ and $\left(\dagger^{\prime}<r\right)$ or $\left(\dagger^{\prime}=r\right)$ with Lemma 5.9.

\section{B. Close to zero.}

Theorem 9.7. If $\mathrm{d}\left(X^{*}\right)+\mathrm{d}(Y)>0$, and $X^{*}$ is split or unramified, then let $\gamma_{\Phi}\left(X^{*}, Y\right)$ and $c_{0}\left(X^{*}\right)$ be as in Definitions 6.5 and 6.10, respectively. Then

$$
M_{X^{*}}^{G}(Y)=c_{0}\left(X^{*}\right)+\frac{2}{n\left(X^{*}\right)} q^{-(r+1)}\left|D_{\mathfrak{g}}(Y)\right|^{-1 / 2} \gamma_{\Phi}\left(X^{*}, Y\right),
$$

where

$$
n\left(X^{*}\right)= \begin{cases}1 & \text { for } X^{*} \text { split } \\ 2 & \text { for } X^{*} \text { elliptic. }\end{cases}
$$


Proof. By (8.10), $m<2$.

By Proposition 8.13, using Notation 8.12, (9.2) becomes

$M_{X^{*}}^{G}(Y)=\frac{1}{2}\left[Q_{3}\left(q^{-1 / 2}\right)+Q_{3}\left(q^{-1 / 2}\right)-q^{-1}-q^{-1} ; 1+\operatorname{sgn}_{\epsilon}+\operatorname{sgn}_{\varpi}+\operatorname{sgn}_{\epsilon \varpi}\right]\left(\theta^{\prime}\right)$.

Since

$$
Q_{3}\left(q^{-1 / 2}\right)+Q_{3}\left(-q^{-1 / 2}\right)=-\left.2 T^{2}\right|_{T=q^{-1 / 2}}=-2 q^{-1},
$$

this simplifies (by the Plancherel formula on $k^{\times} /\left(k^{\times}\right)^{2}$ !) to

$$
M_{X^{*}}^{G}(Y)=\left[-2 q^{-1} ; 2,0,0,0\right] .
$$

Similarly, (9.3) becomes

$$
\begin{aligned}
M_{X^{*}}^{G}(Y)=\frac{1}{2}( & \frac{1}{2}\left(1+\gamma_{\mathrm{un}}(s)\right) \underbrace{\left[Q_{3}\left(q^{-1 / 2}\right)+\gamma_{\mathrm{un}}(s) Q_{3}\left(-q^{-1 / 2}\right) ; 1+\gamma_{\mathrm{un}}(s) \operatorname{sgn}_{\epsilon}\right]}_{(\mathrm{I})} \\
& +\frac{1}{2}\left(1-\gamma_{\mathrm{un}}(s)\right) \underbrace{\left[-\left(1-\gamma_{\mathrm{un}}(s)\right) q^{-1} ;\left(1-\gamma_{\mathrm{un}}(s) \operatorname{sgn}_{\epsilon}\right) \operatorname{sgn}_{\varpi}\right]}_{(\mathrm{II})})\left(\theta^{\prime}\right) .
\end{aligned}
$$

Since $\gamma_{\text {un }}(s)= \pm 1$ (see Definition 6.5), we may replace $\gamma_{\text {un }}(s)$ by 1 in (I) and by -1 in (II), then use (9.8) and check case-by-case to see that the formula simplifies to

$$
M_{X^{*}}^{G}(Y)=\left[-q^{-1} ; 1, \gamma_{\text {un }}(s), 0,0\right]\left(\theta^{\prime}\right) .
$$

\section{Ramified orbital integrals}

Throughout this section, we have

$$
\theta=\varpi, \quad \text { so that } \quad r^{\prime}=r+\frac{1}{2}=: h .
$$

Then $J_{\chi}^{\varpi}=J_{\chi \operatorname{sgn}_{\sigma}}^{\varpi}$ for $\chi \in \widehat{k^{\times}}$, so Proposition 8.11 gives

(10.2) $M_{X^{*}}^{G}(Y)$

$$
=\frac{1}{2}|s|^{-1 / 2}\left(\left(1+\gamma_{\mathrm{ram}}(s)\right) J_{v^{1 / 2}}^{\varpi}(u, v)+\gamma_{\mathrm{un}}(s)\left(1-\gamma_{\mathrm{ram}}(s)\right) J_{v^{1 / 2} \operatorname{sgn}_{\epsilon}}^{\varpi}(u, v)\right) .
$$

By (6.8),

(10.3)

$$
\operatorname{sgn}_{\varpi}(v) G_{\varpi}\left(\Phi_{\varpi^{h+1}}^{\prime}\right)=\operatorname{sgn}_{\varpi}(-\varpi) \gamma_{\mathrm{ram}}(s)=\gamma_{\mathrm{ram}}(s) .
$$

10A. Far from zero. As in Section 9A, the results of this section are special cases of [Waldspurger 1995, Proposition VIII.1].

Theorem 10.4. If $\mathrm{d}\left(X^{*}\right)+\mathrm{d}(Y)<0$ and $X^{*}$ is ramified, then $M_{X^{*}}^{G}(Y)=0$ unless $X^{*}$ and $Y$ lie in $G$-conjugate tori. 
Proof. By (8.10), $m>2$, so Proposition 7.5 and (8.8) show that $M_{X^{*}}^{G}(Y)=0$ unless $\varpi \theta^{\prime} \in\left(k^{\times}\right)^{2}$. By Section 4 , it therefore suffices to consider the case when $-1 \in\left(\mathfrak{f}^{\times}\right)^{2}\left(\operatorname{so~} \operatorname{sgn}_{\varpi}(-1)=1\right)$ and $\theta^{\prime}=\epsilon^{2} \varpi$, that is, $X^{*}$ and $Y$ lie in stably, but not rationally, conjugate tori.

By (8.7), we may take the square root $w$ of $u v$ to be $w=\varpi^{-h} s \epsilon=\epsilon^{-1} u$. Then $u^{-1} w=\epsilon^{-1}$, so Proposition 7.5 shows (whether or not 4 divides $m$ ) that, if $\chi$ is mildly ramified and trivial at -1 , then

$$
J_{\chi \operatorname{sgn}_{\varpi}}(u, v)=\operatorname{sgn}_{\varpi}\left(u^{-1} \varpi\right) J_{\chi}(u, v)=-J_{\chi}(u, v),
$$

hence $J_{\chi}^{\varpi}(u, v)=0$. In particular, this equality holds for $\chi=v^{1 / 2}$ and $\chi=v^{1 / 2} \operatorname{sgn}_{\epsilon}$. It follows from (10.2) that $M_{X^{*}}^{G}(Y)=0$.

Theorem 10.5. If $\mathrm{d}\left(X^{*}\right)+\mathrm{d}(Y)<0$, and $X^{*}$ and $Y$ lie in a common ramified torus $\boldsymbol{T}$ (with $T=\boldsymbol{T}(k)$ ), then

$$
M_{X^{*}}^{G}(Y)=q^{-(h+1)}\left|D_{\mathfrak{g}}(Y)\right|^{-1 / 2} \gamma_{\Phi}\left(X^{*}, Y\right) \sum_{\sigma \in W(G, T)} \Phi\left(\left\langle\operatorname{Ad}^{*}(\sigma)\left(X^{*}\right), Y\right\rangle\right),
$$

where $\gamma_{\Phi}\left(X^{*}, Y\right)$ is as in Definition 6.5.

Proof. Since we have fixed $\theta=\varpi$, the hypothesis implies that $\theta^{\prime}=\varpi$. In particular, $u=v$. Write $\sigma_{\varpi}$ for the nontrivial element of $W\left(\boldsymbol{G}, \boldsymbol{T}_{\varpi}\right)\left(k_{\varpi}\right)$, so that $\operatorname{Ad}^{*}\left(\sigma_{\varpi}\right) X^{*}=-X^{*}$. It is possible that $\sigma_{\varpi}$ is not $k$-rational. More precisely, by Section 4 , we have that

$$
W\left(G, T_{\varpi}\right)= \begin{cases}\left\{1, \sigma_{\varpi}\right\} & \operatorname{sgn}_{\varpi}(-1)=1, \\ \{1\} & \operatorname{sgn}_{\varpi}(-1)=-1 .\end{cases}
$$

By (8.10),

$$
q^{-m / 4}=q^{-(h-\operatorname{ord}(s)) / 2}=q^{-h / 2}|s|^{-1 / 2} .
$$

By Corollary 7.9, since $u=v$,

$$
J_{v^{1 / 2}}^{\varpi}(u, v)=J_{v^{1 / 2} \operatorname{sgn}_{\epsilon}}^{\varpi}(u, v),
$$

so (10.2) becomes

$(\dagger) M_{X^{*}}^{G}(Y)=\frac{1}{2}|s|^{-1 / 2} q^{-(h+1) / 2}\left(\left(1+\gamma_{\mathrm{ram}}(s)\right)+\gamma_{\mathrm{un}}(s)\left(1-\gamma_{\mathrm{ram}}(s)\right)\right) J_{v^{1 / 2}}^{\Phi}(u, v)$.

It remains to compute $J_{v^{1 / 2}}^{\varpi}(u, v)$.

We will use Proposition 7.5, but, for simplicity, we want to avoid splitting into cases depending on whether or not $4 \mid m$. By (8.10), the restrictions to $k \backslash \wp^{h-1}$ of $\frac{1}{2}\left(1+(-1)^{h} \operatorname{sgn}_{\epsilon}\right)=\frac{1}{2}\left(1-\gamma_{\mathrm{un}}\right)$ and $\frac{1}{2}\left(1+\gamma_{\mathrm{un}}\right)$ are characteristic functions that indicate whether $4 \mid m$ or $4 \nmid m$, respectively. (We omit $\wp^{h-1}$ because we are concerned with the case where $\mathrm{d}(Y)<r$, so that $\operatorname{ord}(s)<r-\frac{1}{2}=h-1$.) 
Thus, if $\operatorname{sgn}_{\varpi}(-1)=-1$, then combining Proposition $7.5,(*)$, and (10.3) gives $\left(*_{\mathrm{ns}}\right) \quad J_{v^{\alpha}}(u, v)=q^{-h / 2}|s|^{-1 / 2}$

$$
\begin{aligned}
& \times\left(\frac{1}{2}\left(\left(1-\gamma_{\mathrm{un}}(s)\right)+\left(1+\gamma_{\mathrm{un}}(s)\right) \gamma_{\mathrm{ram}}(s)\right) \times \Phi_{\varpi^{h+1}}^{\prime}\left(2 \varpi^{-h} s\right)\right. \\
& \stackrel{(\S)}{+} \frac{1}{2}\left(\left(1-\gamma_{\mathrm{un}}(s)\right) \stackrel{(\mathrm{II})}{-}\left(1+\gamma_{\mathrm{un}}(s)\right) \gamma_{\mathrm{ram}}(s)\right) \\
\times & \left.\times \Phi_{\varpi^{\prime+1}}^{\prime}\left(-2 \varpi^{-h} s\right)\right) \\
=\frac{1}{2} q^{-(h+1) / 2}\left|s \theta^{\prime}\right|^{-1 / 2} & \times\left(\left(\left(1+\gamma_{\mathrm{ram}}(s)\right)-\gamma_{\mathrm{un}}(s)\left(1-\gamma_{\mathrm{ram}}(s)\right)\right) \Phi\left(\left\langle X^{*}, Y\right\rangle\right)\right. \\
& +\left(\left(1-\gamma_{\mathrm{ram}}(s)\right)-\gamma_{\mathrm{un}}(s)\left(1+\gamma_{\mathrm{ram}}(s)\right)\right) \Phi\left(\left\langle\operatorname{Ad}^{*}\left(\sigma_{\varpi}\right) X^{*}, Y\right\rangle\right)
\end{aligned}
$$

and (changing the sign at (§), but not at (II)) that

$$
\begin{aligned}
J_{\nu^{\alpha} \operatorname{sgn}_{\sigma}}(u, v)=\frac{1}{2} q^{-(h+1) / 2}\left|s \theta^{\prime}\right|^{-1 / 2} & \\
\times & \left(\left(\left(1+\gamma_{\mathrm{ram}}(s)\right)-\gamma_{\mathrm{un}}(s)\left(1-\gamma_{\mathrm{ram}}(s)\right)\right) \Phi\left(\left\langle X^{*}, Y\right\rangle\right)\right. \\
& \left.-\left(\left(1-\gamma_{\mathrm{ram}}(s)\right)-\gamma_{\mathrm{un}}(s)\left(1+\gamma_{\mathrm{ram}}(s)\right)\right) \Phi\left(\left\langle\operatorname{Ad}^{*}\left(\sigma_{\varpi}\right) X^{*}, Y\right\rangle\right)\right),
\end{aligned}
$$

so that

$\left(\ddagger_{\mathrm{ns}}\right) \quad J_{\nu^{\alpha}}^{\Phi}(u, v)$

$$
=\frac{1}{2} q^{-(h+1) / 2}\left|s \theta^{\prime}\right|^{-1 / 2}\left(\left(1+\gamma_{\mathrm{ram}}(s)\right)-\gamma_{\mathrm{un}}(s)\left(1-\gamma_{\mathrm{ram}}(s)\right)\right) \Phi\left(\left\langle X^{*}, Y\right\rangle\right) .
$$

Similarly, if $\operatorname{sgn}_{\varpi}(-1)=1$, then (changing the sign at $(\mathbb{I I})$, but not at $(\S)$, in $\left(*_{\mathrm{ns}}\right)$ ) we obtain

$$
\begin{aligned}
\left(*_{\mathrm{s}}\right) \quad J_{\nu^{\alpha}}(u, v)= & J_{\nu^{\alpha} \operatorname{sgn}_{\varpi}}(u, v) \\
=\frac{1}{2} q^{-(h+1) / 2}\left|s \theta^{\prime}\right|^{-1 / 2} & \left(\left(1+\gamma_{\mathrm{ram}}(s)\right)-\gamma_{\mathrm{un}}(s)\left(1-\gamma_{\mathrm{ram}}(s)\right)\right) \\
& \times\left(\Phi\left(\left\langle X^{*}, Y\right\rangle\right)+\Phi\left(\left\langle\operatorname{Ad}^{*}\left(\sigma_{\varpi}\right) X^{*}, Y\right\rangle\right)\right),
\end{aligned}
$$

so that

$$
\begin{aligned}
J_{v^{\alpha}}^{\varpi}(u, v)= & J_{v^{\alpha}}(u, v) \\
=\frac{1}{2} q^{-(h+1) / 2}\left|s \theta^{\prime}\right|^{-1 / 2}( & \left.\left(1+\gamma_{\mathrm{ram}}(s)\right)-\gamma_{\mathrm{un}}(s)\left(1-\gamma_{\mathrm{ram}}(s)\right)\right) \\
& \times\left(\Phi\left(\left\langle X^{*}, Y\right\rangle\right)+\Phi\left(\left\langle\operatorname{Ad}^{*}\left(\sigma_{\varpi}\right) X^{*}, Y\right\rangle\right)\right) .
\end{aligned}
$$

We may write $\left(\ddagger_{\mathrm{ns}}\right)$ and $\left(\ddagger_{\mathrm{s}}\right)$ uniformly as

$$
\begin{aligned}
J_{v^{\alpha}}^{\varpi}(u, v)=\frac{1}{2} q^{-(h+1) / 2}\left|s \theta^{\prime}\right|^{-1 / 2}((1+ & \left.\left.\gamma_{\mathrm{ram}}(s)\right)-\gamma_{\mathrm{un}}(s)\left(1-\gamma_{\mathrm{ram}}(s)\right)\right) \\
\times & \sum_{\sigma \in N_{G}\left(T_{\varpi}\right) / T_{\varpi}} \Phi\left(\left\langle\operatorname{Ad}^{*}(\sigma) X^{*}, Y\right\rangle\right) .
\end{aligned}
$$


Upon combining $(\dagger),(\ddagger)$, and Lemma 5.9, we obtain the desired formula from

$$
\begin{aligned}
\left(\left(1+\gamma_{\mathrm{ram}}(s)\right)\right. & \left.+\gamma_{\mathrm{un}}(s)\left(1-\gamma_{\mathrm{ram}}(s)\right)\right) \cdot\left(\left(1+\gamma_{\mathrm{ram}}(s)\right)-\gamma_{\mathrm{un}}(s)\left(1-\gamma_{\mathrm{ram}}(s)\right)\right) \\
& =\left(1+\gamma_{\mathrm{ram}}(s)\right)^{2}-\gamma_{\mathrm{un}}(s)^{2}\left(1-\gamma_{\mathrm{ram}}(s)\right)^{2}=4 \gamma_{\mathrm{ram}}(s)=4 \gamma_{\Phi}\left(X^{*}, Y\right)
\end{aligned}
$$

(since $\left.\gamma_{\mathrm{un}}(s)^{2}=1\right)$.

10B. The bad shell. We shall be concerned in this section with the behaviour of $M_{X^{*}}^{G}$ (hence $\hat{\mu}_{X^{*}}^{G}$, by Proposition 11.2) at the "bad shell", that is, on those regular, semisimple elements $Y$ such that $\mathrm{d}(Y)=r$. We assume this is the case throughout the section. By (8.10), this implies that $m=2$ and that $\operatorname{ord}\left(\theta^{\prime}\right)$ is odd, that is, $Y$ belongs to a ramified torus. By Section 4 , we can in fact assume ord $\left(\theta^{\prime}\right)=1$. Then, by Lemma 5.9,

$$
\operatorname{ord}(s)=h-1 \Rightarrow \operatorname{sgn}_{\epsilon}(s)=(-1)^{h-1} \text { and }\left|s \theta^{\prime}\right|=q^{-h} .
$$

By Definition 6.5, the formula that holds in the situation of Theorem 10.9 holds also, suitably understood, in the situation of Theorem 10.8. We find it useful to separate them anyway.

Remark 10.7. In this section only, we need to name the specific ramified torus in which we are interested. We therefore assume in Theorems 10.8 and 10.9 that $X^{*} \in \mathfrak{t}_{\varpi}^{*}$. See Remark 6.9 for a discussion of how to handle other ramified tori.

Theorem 10.8. If $\mathrm{d}\left(X^{*}\right)+\mathrm{d}(Y)=0$, and $Y$ lies in a ramified torus that is not stably conjugate to $\boldsymbol{T}_{\varpi}$, then

$$
M_{X^{*}}^{G}(Y)=\frac{1}{2} q^{-(h+1)} \cdot q^{-1 / 2}\left|D_{\mathfrak{g}}(Y)\right|^{-1 / 2} \sum_{Z \in\left(\mathfrak{t}_{\varpi}\right)_{r: r+}} \Phi\left(\left\langle X^{*}, Z\right\rangle\right) \operatorname{sgn}_{\varpi}\left(Y^{2}-Z^{2}\right),
$$

where we identify the scalar matrices $Y^{2}$ and $Z^{2}$ with elements of $k$ in the natural way.

Proof. By Section 4, it suffices to consider the case where $\theta^{\prime}=\epsilon \varpi$.

By Corollary 7.9, since ord $(u)=\operatorname{ord}(v)$,

$$
J_{v^{1 / 2}}^{\varpi}(u, v)=J_{v^{1 / 2} \operatorname{sgn}_{\epsilon}}^{\varpi}(u, v)
$$

and, by Corollary 7.8 and $(8.8), J_{v^{1 / 2} \operatorname{sgn}_{\sigma}}(u, v)=0$, so

$$
J_{v^{1 / 2}}^{\varpi}(u, v)=\frac{1}{2} J_{v^{1 / 2}}(u, v) .
$$


Hence, by (10.2) and (10.6),

$$
\begin{aligned}
M_{X^{*}}^{G}(Y)= & \frac{1}{4}|s|^{-1 / 2} q^{-(h+1) / 2} \\
& \times\left(\left(1+\gamma_{\mathrm{ram}}(s)\right)-(-1)^{h} \operatorname{sgn}_{\epsilon}(s)\left(1-\gamma_{\mathrm{ram}}(s)\right)\right) J_{v^{1 / 2}}(u, v) \\
= & \frac{1}{4}|s|^{-1 / 2} q^{-(h+1) / 2} \cdot 2 \cdot J_{v^{1 / 2}}(u, v) \\
= & \frac{1}{2}|s|^{-1 / 2} J_{v^{1 / 2}}(u, v) .
\end{aligned}
$$

Finally, another application of Corollary 7.8, together with (8.9), gives that

$$
J_{v^{1 / 2}}(u, v)=q^{-1} \sum_{c \in \wp^{-1} / R} \Phi_{\varpi^{h+1}}^{\prime}(2 c) \operatorname{sgn}_{\varpi}\left(c^{2}-\left(\varpi^{-h} s\right)^{2} \epsilon\right) .
$$

Replacing $c$ by $\varpi^{-h} c$ and using (10.6) again allows us to rewrite

$$
J_{v^{1 / 2}}(u, v)=q^{-(h+2) / 2}\left|s \theta^{\prime}\right|^{-1 / 2} \sum_{c \in \wp^{h-1} / \wp^{h}} \Phi(2 \beta \varpi c) \operatorname{sgn}_{\varpi}\left(c^{2}-s^{2} \epsilon\right) .
$$

By Definition 4.9, the isomorphism $c \mapsto c \cdot \sqrt{\varpi}$ of $k$ with $\mathfrak{t}_{\varpi}$ identifies $\wp^{h-1} / \wp^{h}$ with $\left(\mathfrak{t}_{\varpi}\right)_{(h-1 / 2):(h+1 / 2)}=\left(\mathfrak{t}_{\varpi}\right)_{r: r+}$. If $c$ is mapped to $Z$, then (by (8.5), for example) $2 \beta \varpi c=\left\langle X^{*}, Z\right\rangle$, and

$$
\operatorname{sgn}_{\varpi}\left(c^{2}-s^{2} \epsilon\right)=\operatorname{sgn}_{\varpi}\left(s^{2} \epsilon \varpi-c^{2} \varpi\right)=\operatorname{sgn}_{\varpi}\left(Y^{2}-Z^{2}\right) .
$$

Combining this with $(*),(* *)$, and Lemma 5.9 yields the desired formula.

Theorem 10.9. If $\mathrm{d}\left(X^{*}\right)+\mathrm{d}(Y)=0$, and $\tilde{Y}$ is a stable conjugate of $Y$ that lies in a torus with $X^{*}$, then

$$
\begin{aligned}
M_{X^{*}}^{G}(Y)=\frac{1}{2} q^{-(h+1)}\left|D_{\mathfrak{g}}(Y)\right|^{-1 / 2} & \\
& \times\left(\gamma_{\Phi}\left(X^{*}, Y\right) \sum_{\sigma \in W\left(\boldsymbol{G}, \boldsymbol{T}_{\varpi}\right)} \Phi\left(\left\langle\operatorname{Ad}^{*}(\sigma) X^{*}, \tilde{Y}\right\rangle\right)\right. \\
& \left.+q^{-1 / 2} \sum_{\substack{Z \in\left(\mathfrak{t}_{\varpi}\right)_{r, r+} \\
Z \neq \pm(\tilde{Y}}} \Phi\left(\left\langle X^{*}, Z\right\rangle\right) \operatorname{sgn}_{\varpi}\left(Y^{2}-Z^{2}\right)\right),
\end{aligned}
$$

where $\gamma_{\Phi}\left(X^{*}, Y\right)$ is as in Definition 6.5.

Proof. Implicit in the statement is the hypothesis that $\mathfrak{t}=\mathfrak{t}_{\theta^{\prime}}$ is stably conjugate to $\mathfrak{t}_{\varpi}$, so that, by Section 4, we have $\theta^{\prime}=x^{2} \varpi$ for some $x \in R^{\times}$. The proof proceeds much as in Theorem 10.8.

By (10.6) and Corollary 7.9, since ord $(u)=\operatorname{ord}(v),(10.2)$ becomes

$$
M_{X^{*}}^{G}(Y)=|s|^{-1 / 2} q^{-(h+1) / 2} J_{v^{1 / 2}}^{\varpi}(u, v) .
$$

By (8.7), we may take the square root $w$ of $u v$ to be $w=\varpi^{-h} x s$. 
Combining Corollary 7.8 with (8.7), (8.9), and (10.6) gives

$$
\begin{aligned}
J_{v^{\alpha}}(u, v) & =q^{-1} \sum_{\substack{c \in \wp^{-1} / R \\
c \neq \pm \varpi^{-h} x s}} \Phi_{\varpi^{h+1}}^{\prime}(2 c) \operatorname{sgn}_{\varpi}\left(c^{2}-\left(\varpi^{-h} x s\right)^{2}\right) \\
& =q^{-1} \sum_{\substack{c \in \wp^{h-1} / \wp^{h} \\
c \neq \pm \pm s}} \Phi(2 \beta \varpi c) \operatorname{sgn}_{\varpi}\left(c^{2}-x^{2} s^{2}\right) \\
& =q^{-(h+2) / 2\left|s \theta^{\prime}\right|^{-1 / 2}} \sum_{\substack{c \in \wp^{h-1} / \wp^{h} \\
c \neq \pm x s}} \Phi(2 \beta \varpi c) \operatorname{sgn}_{\varpi}\left(c^{2}-x^{2} s^{2}\right) .
\end{aligned}
$$

Note that $Y^{2}=s^{2} \theta^{\prime}=x^{2} s^{2} \varpi$, and that

$$
\widetilde{Y}:=x s \sqrt{\varpi}=\operatorname{Ad}\left(\begin{array}{cc}
\sqrt{x} & 0 \\
0 & \sqrt{x}-1
\end{array}\right) Y
$$

is a stable conjugate of $Y$ that lies in $\mathfrak{t}_{\varpi}$. (Here, $\sqrt{\varpi}$ is an element of $\mathfrak{g}$, but $\sqrt{x}$ is an element of an extension field of $k$.) As in Theorem 10.8, if $Z=c \cdot \sqrt{\varpi}$, then $\left\langle X^{*}, Z\right\rangle=2 \beta \varpi c$ and $\operatorname{sgn}_{\varpi}\left(c^{2}-x^{2} s^{2}\right)=\operatorname{sgn}_{\varpi}\left(Y^{2}-Z^{2}\right)$. That is, upon using again the bijection $\wp^{h-1} / \wp^{h} \rightarrow\left(\mathfrak{t}_{\varpi}\right)_{r: r+}$ given by $c \mapsto c \cdot \sqrt{\varpi}$, we obtain

$$
\left(* *_{1}\right) \quad J_{v^{1 / 2}}(u, v)=q^{-(h+2) / 2}\left|s \theta^{\prime}\right|^{-1 / 2} \sum_{\substack{Z \in\left(\mathfrak{t}_{\sigma}\right)_{r: x^{+}} \\ Z \neq 0, \pm \widetilde{Y}}} \Phi\left(\left\langle X^{*}, Z\right\rangle\right) \operatorname{sgn}_{\varpi}\left(Y^{2}-Z^{2}\right) \text {. }
$$

Similarly, combining Corollary 7.8 with (8.9), Lemma 5.9, and (10.3) gives

$$
\begin{aligned}
\left(* *_{\varpi}\right) \quad J_{\nu^{1 / 2} \operatorname{sgn}_{\varpi}}(u, v) & =q^{-1 / 2} \gamma_{\operatorname{ram}}(s)(\Phi(2 \beta \varpi x s)+\Phi(-2 \beta \varpi x s)) \\
& =q^{-(h+1) / 2}\left|s \theta^{\prime}\right|^{-1 / 2} \gamma_{\operatorname{ram}}(s) \sum_{\sigma \in W(\boldsymbol{G}, \boldsymbol{T})} \Phi\left(\left\langle\operatorname{Ad}^{*}(\sigma) X^{*}, \tilde{Y}\right\rangle\right) .
\end{aligned}
$$

Combining $(*),\left(* *_{1}\right),\left(* *_{\varpi}\right)$, and Lemma 5.9 gives the desired formula.

10C. Close to zero.

Theorem 10.10. If $\mathrm{d}\left(X^{*}\right)+\mathrm{d}(Y)>0$, and $X^{*}$ is ramified, then let $\gamma_{\Phi}\left(X^{*}, Y\right)$ and $c_{0}\left(X^{*}\right)$ be as in Definitions 6.5 and 6.10, respectively. Then

$$
M_{X^{*}}^{G}(Y)=c_{0}\left(X^{*}\right)+q^{-(h+1)}\left|D_{\mathfrak{g}}(Y)\right|^{-1 / 2} \gamma_{\Phi}\left(X^{*}, Y\right) .
$$

Proof. By (8.10), $m<2$.

By Proposition 8.13 and (6.7), using Notation 8.12 changes (10.2) into

$$
\begin{aligned}
& M_{X^{*}}^{G}(Y) \\
& =\frac{1}{2}\left(\frac{1}{2}\left(1+\gamma_{\mathrm{ram}}(s)\right)\left[Q_{3}\left(q^{-1 / 2}\right)+\gamma_{\mathrm{ram}}(s) q^{-1} ; 1+\gamma_{\mathrm{ram}}(s)^{-1} \operatorname{sgn}_{\varpi}\right]\right. \\
& \left.\quad+\frac{1}{2}\left(1-\gamma_{\mathrm{ram}}(s)\right) \times\left[-Q_{3}\left(-q^{-1 / 2}\right)+\gamma_{\mathrm{ram}}(s) q^{-1} ;\left(1-\gamma_{\mathrm{ram}}(s)^{-1} \operatorname{sgn}_{\varpi}\right) \operatorname{sgn}_{\epsilon}\right]\right)\left(\theta^{\prime}\right) .
\end{aligned}
$$


By (9.8) and the fact that

$$
Q_{3}\left(q^{-1 / 2}\right)-Q_{3}\left(-q^{-1 / 2}\right)=-\left.2 T\left(T^{2}+1\right)\right|_{T=q^{-1 / 2}}=-2 q^{-3 / 2}(q+1),
$$

we may check case-by-case to see that this simplifies to

$$
M_{X^{*}}^{G}(Y)=\left[-\frac{1}{2} q^{-3 / 2}(q+1) ; 1,0, \gamma_{\mathrm{ram}}(s), 0\right]\left(\theta^{\prime}\right) .
$$

\section{An integral formula}

Our efforts so far have focused on computing the function $M_{X^{*}}^{G}$ of Definition 8.4, whereas we are really interested in the function $\hat{\mu}_{X^{*}}^{G}$ of Notation 5.7. We can now show that they are actually equal.

Lemma 11.1. If $f \in L^{1}(G)$, then

$$
\int_{G} f(g) d g=\int_{k_{\theta}^{\times}} \int_{k} f\left(\varphi_{\theta}^{-1}(\alpha, t)\right) \mathrm{d} t \mathrm{~d}^{\times} \alpha .
$$

In Lemma 11.1, $\mathrm{d} g, \mathrm{~d} t$, and $\mathrm{d}^{\times} \alpha$ are Haar measures on the obvious groups. Given any two of them, the third can be chosen so that the identity is satisfied. Since Definition 5.4 requires a measure on $G / C_{G}\left(X^{*}\right)$, not on $G$, we do not worry much here about normalisations (although a specific one is used in the proof).

Proof. With respect to the coordinate charts

$$
(a, b, c) \mapsto\left(\begin{array}{cc}
a & b \\
c & (1+b c) / a
\end{array}\right)
$$

(for $a \neq 0$ ) on $G$ and

$$
(a, b, t) \mapsto(a+b \sqrt{\theta}, t)
$$

on $k_{\theta}^{\times} \times k$, the Jacobian of $\varphi_{\theta}$ at

$$
g=\left(\begin{array}{ll}
a & b \\
c & d
\end{array}\right)
$$

(with $a \neq 0$ ) is $a^{-1} N_{\theta}(\alpha)$, where $\varphi_{\theta}(g)=(\alpha, t)$.

In particular, the Haar measure

$$
|a|^{-1} \mathrm{~d} a \mathrm{~d} b \mathrm{~d} c
$$

on $G$ is carried to the measure

$$
\left|N_{\theta}(a+b \sqrt{\theta})\right|^{-1} \mathrm{~d} a \mathrm{~d} b \mathrm{~d} t=\left|N_{\theta}(\alpha)\right|^{-1} \mathrm{~d} \alpha \mathrm{d} t=\mathrm{d}^{\times} \alpha \mathrm{d} t
$$

on $k_{\theta}^{\times} \times k$, as desired. 
Proposition 11.2. If $X^{*} \in \mathfrak{g}^{*}$ and $Y \in \mathfrak{g}$ are regular and semisimple, then

$$
\hat{\mu}_{X^{*}}^{G}(Y)=M_{X^{*}}^{G}(Y),
$$

where $M_{X^{*}}^{G}$ is as in Definition 8.4, and the Haar measure $\mathrm{d} \dot{g}$ on $G / C_{G}\left(X^{*}\right)$ of Notation 5.3 is normalised so that

$$
\operatorname{meas}_{\mathrm{d} \dot{g}}(\dot{K})= \begin{cases}q^{-1}(q+1) & \text { for } X^{*} \text { split } \\ q^{-1}(q-1) & \text { for } X^{*} \text { unramified } \\ \frac{1}{2} q^{-2}\left(q^{2}-1\right) & \text { for } X^{*} \text { ramified }\end{cases}
$$

where $\dot{K}$ is the image in $G / C_{G}\left(X^{*}\right)$ of $\mathrm{SL}_{2}(R)$.

Proof. We will maintain Notation 5.1. In particular, $X^{*} \in \mathfrak{t}_{\theta}^{*}$.

By the explicit formulae of the previous sections (specifically, Theorems 9.5, 9.6, 9.7, 10.4, 10.5, 10.8, 10.9, and 10.10), $M_{X^{*}}^{G} \in C^{\infty}\left(\mathfrak{g}^{\text {rss }}\right)$. This result plays the role of [Adler and DeBacker 2004, Corollary A.3.4]; we now imitate the proof of [ibid., Theorem A.1.2].

If $f \in C_{c}\left(\mathfrak{g}^{\text {rss }}\right)$, then there is a lattice $\mathscr{L} \subseteq \mathfrak{g}$ such that $f \cdot M_{X^{*}}^{G}$ is invariant under translation by $\mathscr{L}$. Then

$$
\int_{\mathfrak{g}} f(Y) M_{X^{*}}^{G}(Y) \mathrm{d} Y=\operatorname{meas}_{\mathrm{d} Y}(\mathscr{L}) \sum_{Y \in \mathfrak{g} / \mathscr{L}} f(Y) \cdot \oint_{k_{\theta}^{\times} / C_{\theta}} \oint_{k} \Phi\left(\left\langle X^{*}, Y\right\rangle_{\alpha, t}\right) \mathrm{d} t \mathrm{~d}^{\times} \dot{\alpha} .
$$

Since the sum is finitely supported, we may bring it inside the integral. By (8.5) and Definition 5.5,

$$
\begin{array}{rl}
\int_{\mathfrak{g}} & f(Y) M_{X^{*}}^{G}(Y) \mathrm{d} Y \\
= & \oint_{k_{\theta}^{\times} / C_{\theta}} \oint_{k} \operatorname{meas}_{\mathrm{d} Y}(\mathscr{L}) \sum_{Y \in \mathfrak{g} / \mathscr{L}} f(Y) \Phi\left(\left\langle\operatorname{Ad}^{*}\left(\varphi_{\theta}^{-1}(\alpha, t)\right) X^{*}, Y\right\rangle\right) \mathrm{d} t \mathrm{~d}^{\times} \dot{\alpha} \\
= & \oint_{k_{\theta}^{\times} / C_{\theta}} \oint_{k} \int_{\mathfrak{g}} f(Y) \Phi\left(\left\langle\operatorname{Ad}^{*}\left(\varphi_{\theta}^{-1}(\alpha, t)\right) X^{*}, Y\right\rangle\right) \mathrm{d} Y \mathrm{~d} t \mathrm{~d}^{\times} \dot{\alpha} \\
=\oint_{k_{\theta}^{\times} / C_{\theta}} \oint_{k} \hat{f}\left(\operatorname{Ad}^{*}\left(\varphi_{\theta}^{-1}(\alpha, t)\right) X^{*}\right) \mathrm{d} t \mathrm{~d}^{\times} \dot{\alpha},
\end{array}
$$

where $\varphi_{\theta}$ is as in Definition 8.2.

On the other hand, again by Definition 5.5,

$\hat{\mu}_{X^{*}}^{G}(f):=\mu_{X^{*}}^{G}(\hat{f})=\int_{G / T_{\theta}} \hat{f}\left(\operatorname{Ad}^{*}(g) X^{*}\right) \mathrm{d} \dot{g}=\int_{\bar{U} \backslash G / T_{\theta}} \int_{\bar{U}} \hat{f}\left(\operatorname{Ad}^{*}(\bar{u} g) X^{*}\right) \mathrm{d} \bar{u} \mathrm{~d} \ddot{g}$,

where

$$
\bar{U}=\left\{\left(\begin{array}{ll}
1 & 0 \\
b & 1
\end{array}\right): b \in k\right\}
$$


By Lemmata 11.1 and 8.3 , and $(*)$, if $\mathrm{d} \dot{g}$ is properly normalised, then

$$
\hat{\mu}_{X^{*}}^{G}(f)=\int_{k_{\theta}^{\times} / C_{\theta}} \int_{k} \hat{f}\left(\operatorname{Ad}^{*}\left(\varphi_{\theta}^{-1}(\alpha, t)\right) X^{*}\right) \mathrm{d} t \mathrm{~d}^{\times} \dot{\alpha}=\int_{\mathfrak{g}} f(Y) M_{X^{*}}^{G}(Y) \mathrm{d} Y .
$$

It remains only to compute the normalisation of $\mathrm{d} \dot{g}$. We do so case-by-case. If $X^{*}$ is split, so that we may take $\theta=1$, then the image under $\varphi_{1}$ of

$$
\left(1+\wp_{1}\right) \times \wp \subseteq k_{1}^{\times} \times k
$$

is precisely the kernel $K_{+}$of the (component-wise) reduction map $\mathrm{SL}_{2}(R) \rightarrow$ $\mathrm{SL}_{2}(\mathfrak{f})$. Here, we have written $1+\wp_{1}=\left\{(a, b) \in k_{1}: a \in 1+\wp, b \in \wp\right\}$. Thus,

$$
\left(1+\wp_{1}\right) C_{1} / C_{1} \times \wp \stackrel{\sim}{\rightarrow} K_{+} T_{1} / T_{1}
$$

Now $N_{1}: 1+\wp_{1} \rightarrow 1+\wp$ is surjective, so by Definitions 2.1 and 8.4, the measure (in $k_{1} / C_{1} \times k$ ) of the domain is

$$
\operatorname{meas}_{\mathrm{d}^{\times} x}(1+\wp) \cdot \operatorname{meas}_{\mathrm{d} x}(\wp)=q^{-2} .
$$

Since $\dot{K}=\mathrm{SL}_{2}(R) T_{1} / T_{1}$ is tiled by

$$
\left[\mathrm{SL}_{2}(R) T_{1}: K_{+} T_{1}\right]=\left[\mathrm{SL}_{2}(R): K_{+}\left(T_{1} \cap \mathrm{SL}_{2}(R)\right)\right]=\left[\mathrm{SL}_{2}(\mathfrak{f}): \mathrm{T}_{1}(\mathfrak{f})\right]=q(q+1)
$$

copies of $K_{+} T_{1} / T_{1}$, where

$$
\mathrm{T}_{1}:=\left\{\left(\begin{array}{ll}
a & b \\
b & a
\end{array}\right): a^{2}-b^{2}=1\right\}
$$

we see that, in this case, $\mathrm{d} \dot{g}$ assigns $\dot{K}$ measure $q^{-2} \cdot q(q+1)=q^{-1}(q+1)$.

The remaining cases are easier, since $C_{\theta}$ is contained in the ring $R_{\theta}$ of integers in $k_{\theta}$, and (for our choices of $\theta$ ) $T_{\theta}$ is contained in $\operatorname{SL}_{2}(R)$. If $X^{*}$ is unramified, so that we may take $\theta=\epsilon$, then the image under $\varphi_{\epsilon}$ of $R_{\epsilon}^{\times} \times R$ is precisely $\operatorname{SL}_{2}(R)$. Since $N_{\epsilon}: R_{\epsilon}^{\times} \rightarrow R^{\times}$is surjective, we see that, in this case, $\mathrm{d} \dot{g}$ assigns $\dot{K}$ measure $\operatorname{meas}_{\mathrm{d}^{\times}{ }_{x}}\left(R_{\epsilon}^{\times}\right) \cdot \operatorname{meas}_{\mathrm{d} x}(R)=q^{-1}(q-1)$.

If $X^{*}$ is ramified, so that we may take $\theta=\varpi$, then the image under $\varphi_{\varpi}$ of $R_{\varpi}^{\times} \times \wp$ is precisely the Iwahori subgroup $\mathscr{I}$, that is, the preimage in $\mathrm{SL}_{2}(R)$ of

$$
\mathrm{B}(\mathfrak{f}):=\left\{\left(\begin{array}{ll}
a & b \\
0 & a
\end{array}\right): a \in \mathfrak{f}^{\times}, b \in \mathfrak{f}\right\}
$$

under the reduction map $\mathrm{SL}_{2}(R) \rightarrow \mathrm{SL}_{2}(\mathfrak{f})$. Since $N_{\varpi}: R_{\varpi}^{\times} \rightarrow R^{\times}$has cokernel of order 2 , we see that, in this case, $\mathrm{d} \dot{g}$ assigns $\dot{K}$ measure

$$
\frac{1}{2} \operatorname{meas}_{\mathrm{d}^{\times} x}\left(R^{\times}\right) \cdot \operatorname{meas}_{\mathrm{d} x}(\wp) \cdot\left[\mathrm{SL}_{2}(\mathfrak{f}): \mathrm{B}\right]=\frac{1}{2} q^{-2}\left(q^{2}-1\right) .
$$


Thus, all the results we have proven for $M_{X^{*}}^{G}$ are actually results about $\hat{\mu}_{X^{*}}^{G}$. We close by summarising some results that can be stated in a fairly uniform fashion (that is, mostly independent of the "type" of $X^{*}$, in the sense of Definition 4.4). This theorem does not cover everything we have shown about Fourier transforms of semisimple orbital integrals (in particular, it says nothing about the behaviour of ramified orbital integrals on the "bad shell", as in Section 10B); for that, the reader should refer to the detailed results of Sections 9-10.

Theorem 11.3. If $\mathrm{d}\left(X^{*}\right)+\mathrm{d}(Y)<0\left(\right.$ or $\mathrm{d}\left(X^{*}\right)+\mathrm{d}(Y) \leq 0$ and $X^{*}$ is split or unramified), then

$$
\hat{\mu}_{X^{*}}^{G}(Y)=q^{-\left(r^{\prime}+1\right)}\left|D_{\mathfrak{g}}(Y)\right|^{-1 / 2} \gamma_{\Phi}\left(X^{*}, Y\right) \sum_{\sigma \in W(G, T)} \Phi\left(\left\langle\operatorname{Ad}^{*}(\sigma) X^{*}, Y\right\rangle\right)
$$

if $X^{*}$ and $Y$ lie in a common torus $\boldsymbol{T}$ (with $T=\boldsymbol{T}(k)$ ), and

$$
\hat{\mu}_{X^{*}}^{G}(Y)=0
$$

if $X^{*}$ and $Y$ do not lie in G-conjugate tori. Here, $r^{\prime}$ is as in Notation 5.2, and $\gamma_{\Phi}\left(X^{*}, Y\right)$ is as in Definition 6.5.

If $\mathrm{d}\left(X^{*}\right)+\mathrm{d}(Y)>0$, then

$$
\hat{\mu}_{X^{*}}^{G}(Y)=c_{0}\left(X^{*}\right)+q^{-\left(r^{\prime}+1\right)}\left|D_{\mathfrak{g}}(Y)\right|^{-1 / 2} \gamma_{\Phi}\left(X^{*}, Y\right) .
$$

Here, $\gamma_{\Phi}\left(X^{*}, Y\right)$ and $c_{0}\left(X^{*}\right)$ are as in Definitions 6.5 and 6.10, respectively.

Proof. This is an amalgamation of parts of Theorems 9.5, 9.6, 9.7, 10.4, 10.5, and 10.10 and Proposition 11.2.

\section{Acknowledgements}

This paper, and the paper [Adler et al. 2011] that follows it, would not have been possible without the advice and guidance of Paul J. Sally, Jr. It is a pleasure to thank him, as well as Stephen DeBacker and Jeffrey D. Adler, both of whom offered useful suggestions regarding this paper.

\section{References}

[Adler 1998] J. D. Adler, "Refined anisotropic $K$-types and supercuspidal representations", Pacific J. Math. 185:1 (1998), 1-32. MR 2000f:22019 Zbl 0924.22015

[Adler and DeBacker 2002] J. D. Adler and S. M. DeBacker, "Some applications of Bruhat-Tits theory to harmonic analysis on the Lie algebra of a reductive p-adic group", Michigan Math. J. 50:2 (2002), 263-286. MR 2003g:22016 Zbl 1018.22013

[Adler and DeBacker 2004] J. D. Adler and S. M. DeBacker, "Murnaghan-Kirillov theory for supercuspidal representations of tame general linear groups", J. Reine Angew. Math. 575 (2004), 1-35. MR 2005j:22008 Zbl 1054.22014 
[Adler and Spice 2009] J. D. Adler and L. R. Spice, "Supercuspidal characters of reductive $p$-adic groups", Amer. J. Math. 131:4 (2009), 1137-1210. MR 2011a:22018 Zbl 1173.22012 arXiv 0707. 3313

[Adler et al. 2011] J. D. Adler, S. M. DeBacker, P. J. Sally, Jr., and L. R. Spice, "Supercuspidal characters of $\mathrm{SL}_{2}$ over a $p$-adic field", pp. 19-69 in Harmonic analysis on reductive, p-adic groups (San Francisco, CA, 2010), edited by R. S. Doran et al., Contemp. Math. 543, Amer. Math. Soc., Providence, RI, 2011. MR 2798422 Zbl 1222.22007

[Baruch 1997] E. M. Baruch, "On Bessel distributions for $\mathrm{GL}_{2}$ over a p-adic field", J. Number Theory 67:2 (1997), 190-202. MR 98k:22042 Zbl 0887.22023

[Baruch 2001] E. M. Baruch, "On Bessel distributions for quasi-split groups", Trans. Amer. Math. Soc. 353:7 (2001), 2601-2614. MR 2002b:22029 Zbl 0976.22015

[Baruch 2003] E. M. Baruch, "Bessel functions for GL(3) over a p-adic field", Pacific J. Math. 211:1 (2003), 1-34. MR 2004k:22022 Zbl 1055.22013

[Baruch 2004] E. M. Baruch, "Bessel distributions for GL(3) over the p-adics", Pacific J. Math. 217:1 (2004), 11-27. MR 2005h:22025 Zbl 1064.22008

[Baruch 2005] E. M. Baruch, "Bessel functions for GL(n) over a p-adic field", pp. 1-40 in Automorphic representations, L-functions and applications: progress and prospects (Columbus, $\mathrm{OH}, 2003$ ), edited by J. W. Cogdell et al., Ohio State Univ. Math. Res. Inst. Publ. 11, de Gruyter, Berlin, 2005. MR 2007d:22011 Zbl 1103.22006

[Bushnell and Kutzko 1993a] C. J. Bushnell and P. C. Kutzko, The admissible dual of GL(N) via compact open subgroups, Annals of Mathematics Studies 129, Princeton University Press, Princeton, NJ, 1993. MR 94h:22007 Zbl 0787.22016

[Bushnell and Kutzko 1993b] C. J. Bushnell and P. C. Kutzko, "The admissible dual of SL(N), I", Ann. Sci. École Norm. Sup. (4) 26:2 (1993), 261-280. MR 94a:22033 Zbl 0787.22017

[Bushnell and Kutzko 1994] C. J. Bushnell and P. C. Kutzko, "The admissible dual of SL(N), II", Proc. London Math. Soc. (3) 68:2 (1994), 317-379. MR 94k:22035 Zbl 0801.22011

[Corwin 1989] L. Corwin, "The unitary dual for the multiplicative group of arbitrary division algebras over local fields", J. Amer. Math. Soc. 2:3 (1989), 565-598. MR 90d:22021 Zbl 0706.22013

[Corwin et al. 1995] L. Corwin, A. Moy, and P. J. Sally, Jr., "Supercuspidal character formulas for GL $\ell$ ", pp. 1-11 in Representation theory and harmonic analysis: conference in honor of Ray A. Kunze (Cincinnati, OH, 1994), edited by T. Ton-That et al., Contemp. Math. 191, Amer. Math. Soc., Providence, RI, 1995. MR 96m:22037 Zbl 0839.22022

[Cunningham and Gordon 2009] C. Cunningham and J. Gordon, "Motivic proof of a character formula for SL(2)”, Experiment. Math. 18:1 (2009), 11-44. MR 2010i:22014 Zbl 1179.22018

[DeBacker 1997] S. M. DeBacker, On supercuspidal characters of $\mathrm{GL}_{\ell}, \ell$ a prime, thesis, The University of Chicago, 1997.

[DeBacker 2002] S. M. DeBacker, "Homogeneity results for invariant distributions of a reductive $p$ adic group", Ann. Sci. École Norm. Sup. (4) 35:3 (2002), 391-422. MR 2003i:22019 Zbl 0999.22013

[DeBacker and Reeder 2009] S. M. DeBacker and M. Reeder, "Depth-zero supercuspidal L-packets and their stability", Ann. of Math. (2) 169:3 (2009), 795-901. MR 2010d:22023 Zbl 1193.11111

[DeBacker and Sally 2000] S. M. DeBacker and P. J. Sally, Jr., "Germs, characters, and the Fourier transforms of nilpotent orbits", pp. 191-221 in The mathematical legacy of Harish-Chandra (Baltimore, MD, 1998), edited by R. S. Doran and V. S. Varadarajan, Proc. Sympos. Pure Math. 68, Amer. Math. Soc., Providence, RI, 2000. MR 2001i:22022 Zbl 0960.22017

[Dwork 1974] B. Dwork, "Bessel functions as p-adic functions of the argument", Duke Math. J. 41:4 (1974), 711-738. MR 52 \#8124 Zbl 0302.14008 
[Gindikin and Karpelevič 1962] S. G. Gindikin and F. I. Karpelevič, "Plancherel measure for symmetric Riemannian spaces of non-positive curvature”, Dokl. Akad. Nauk SSSR 145 (1962), 252-255. In Russian; translated in Sov. Math 3 (1962), 962-965. MR 27 \#240 Zbl 0168.27503

[Hales 1994] T. C. Hales, "Hyperelliptic curves and harmonic analysis: why harmonic analysis on reductive $p$-adic groups is not elementary", pp. 137-169 in Representation theory and analysis on homogeneous spaces (New Brunswick, NJ, 1993), edited by S. Gindikin et al., Contemp. Math. 177, Amer. Math. Soc., Providence, RI, 1994. MR 96d:22024 Zbl 0834.22017

[Harish-Chandra 1966] Harish-Chandra, "Discrete series for semisimple Lie groups, II: Explicit determination of the characters", Acta Math. 116:1 (1966), 1-111. MR 36 \#2745 Zbl 0199.20102

[Harish-Chandra 1970] Harish-Chandra, Harmonic analysis on reductive p-adic groups, Lecture Notes in Mathematics 162, Springer, Berlin, 1970. MR 54 \#2889 Zbl 0202.41101

[Harish-Chandra 1999] Harish-Chandra, Admissible invariant distributions on reductive p-adic groups, University Lecture Series 16, Amer. Math. Soc., Providence, RI, 1999. MR 2001b:22015 Zbl 0928.22017

[Howe 1971] R. E. Howe, "Representation theory for division algebras over local fields: tamely ramified case", Bull. Amer. Math. Soc. 77:6 (1971), 1063-1066. MR 44 \#4146 Zbl 0223.22009

[Howe 1973] R. E. Howe, "Two conjectures about reductive p-adic groups", pp. 377-380 in Harmonic analysis on homogeneous spaces (Williamstown, MA, 1972), edited by C. C. Moore, Proc. Sympos. Pure Math. 26, Amer. Math. Soc., Providence, RI, 1973. MR 49 \#3044 Zbl 0284.22004

[Kim and Murnaghan 2003] J.-L. Kim and F. Murnaghan, "Character expansions and unrefined minimal K-types”, Amer. J. Math. 125:6 (2003), 1199-1234. MR 2004k:22024 Zbl 1037.22035

[Kim and Murnaghan 2006] J.-L. Kim and F. Murnaghan, " $K$-types and $\Gamma$-asymptotic expansions", J. Reine Angew. Math. 592 (2006), 189-236. MR 2007m:22016 Zbl 1089.22011

[Langlands 1979] R. P. Langlands, "Stable conjugacy: definitions and lemmas", Canad. J. Math. 31:4 (1979), 700-725. MR 82j:10054 Zbl 0421.12013

[Langlands 2011] R. P. Langlands, "Singularités et transfert", 2011, Available at http://publications. ias.edu/node/139. To appear in Annales des Sciences Mathématiques du Québec.

[Lidl and Niederreiter 1997] R. Lidl and H. Niederreiter, Finite fields, 2nd ed., Encyclopedia of Mathematics and its Applications 20, Cambridge University Press, Cambridge, 1997. MR 97i: 11115 Zbl 0866.11069

[Morris 1992] L. Morris, "Tamely ramified supercuspidal representations of classical groups, II: Representation theory”, Ann. Sci. École Norm. Sup. (4) 25:3 (1992), 233-274. MR 93h:22032 Zbl 0782.22012

[Moy 1986] A. Moy, "Local constants and the tame Langlands correspondence", Amer. J. Math. 108:4 (1986), 863-930. MR 88b:11081 Zbl 0597.12019

[Moy and Prasad 1994] A. Moy and G. Prasad, "Unrefined minimal $K$-types for $p$-adic groups", Invent. Math. 116:1 (1994), 393-408. MR 95f:22023 Zbl 0804.22008

[Moy and Prasad 1996] A. Moy and G. Prasad, "Jacquet functors and unrefined minimal $K$-types", Comment. Math. Helv. 71:1 (1996), 98-121. MR 97c:22021 Zbl 0860.22006

[Murnaghan 1995a] F. Murnaghan, "Characters of supercuspidal representations of SL(n)", Pacific J. Math. 170:1 (1995), 217-235. MR 96k:22030 Zbl 0848.22022

[Murnaghan 1995b] F. Murnaghan, "Local character expansions for supercuspidal representations of U(3)”, Canad. J. Math. 47:3 (1995), 606-640. MR 96i:22026 Zbl 0909.22030

[Murnaghan 1996a] F. Murnaghan, "Characters of supercuspidal representations of classical groups", Ann. Sci. École Norm. Sup. (4) 29:1 (1996), 49-105. MR 98c:22016 Zbl 0856.22025 
[Murnaghan 1996b] F. Murnaghan, "Local character expansions and Shalika germs for GL(n)", Math. Ann. 304:1 (1996), 423-455. MR 98b:22020 Zbl 0841.22008

[Murnaghan 2000] F. Murnaghan, "Germs of characters of admissible representations", pp. 501515 in The mathematical legacy of Harish-Chandra (Baltimore, MD, 1998), edited by R. S. Doran and V. S. Varadarajan, Proc. Sympos. Pure Math. 68, Amer. Math. Soc., Providence, RI, 2000. MR 2001i:22023 Zbl 0957.22020

[Sally and Shalika 1968] P. J. Sally, Jr. and J. A. Shalika, "Characters of the discrete series of representations of SL(2) over a local field", Proc. Nat. Acad. Sci. USA 61:4 (1968), 1231-1237. MR 38 \#5994 Zbl 0198.18203

[Sally and Shalika 1969] P. J. Sally, Jr. and J. A. Shalika, "The Plancherel formula for SL(2) over a local field”, Proc. Nat. Acad. Sci. USA 63:3 (1969), 661-667. MR 51 \#813 Zbl 0201.17703

[Sally and Shalika 1984] P. J. Sally, Jr. and J. A. Shalika, "The Fourier transform of orbital integrals on $\mathrm{SL}_{2}$ over a $p$-adic field", pp. 303-340 in Lie group representations, II (College Park, MD, 1982/1983), edited by R. Herb et al., Lecture Notes in Math. 1041, Springer, Berlin, 1984. MR 86a:22017 Zbl 0542.22017

[Sally and Spice 2009] P. J. Sally, Jr. and L. R. Spice, "Character theory or reductive $p$-adic groups", pp. 103-111 in Ottawa lectures on admissible representations of reductive p-adic groups, edited by C. Cunningham and M. Nevins, Fields Inst. Monogr. 26, Amer. Math. Soc., Providence, RI, 2009. MR 2010b:22012 Zbl 1176.22016

[Sally and Taibleson 1966] P. J. Sally, Jr. and M. H. Taibleson, "Special functions on locally compact fields", Acta Math. 116:1 (1966), 279-309. MR 34 \#6168 Zbl 0173.07005

[Schmid 1968] W. Schmid, "Homogeneous complex manifolds and representations of semisimple Lie groups”, Proc. Nat. Acad. Sci. USA 59:1 (1968), 56-59. MR 37 \#1520 Zbl 0164.15803

[Shalika 2004] J. A. Shalika, "Representation of the two by two unimodular group over local fields", pp. 1-38 in Contributions to automorphic forms, geometry, and number theory (Baltimore, MD, 2002), edited by H. Hida et al., Johns Hopkins University Press, Baltimore, MD, 2004. MR 2005f: 22028 Zbl 1076.11032

[Spice 2005] L. R. Spice, "Supercuspidal characters of SL $l$ over a $p$-adic field, $l$ a prime", Amer. $J$. Math. 127:1 (2005), 51-100. MR 2005k:22028 Zbl 1059.22020

[Stevens 2008] S. Stevens, "The supercuspidal representations of $p$-adic classical groups", Invent. Math. 172:2 (2008), 289-352. MR 2010e:22008 Zbl 1140.22016 arXiv math.RT/0607622

[Takahashi 2003] T. Takahashi, "Formulas for tamely ramified supercuspidal characters of GL 3 ", Trans. Amer. Math. Soc. 355:2 (2003), 567-591. MR 2003i:22020 Zbl 1033.22005

[Takahashi 2005] T. Takahashi, "On some constants in the supercuspidal characters of $\mathrm{GL}_{l}, l$ a prime $\neq$ p", Trans. Amer. Math. Soc. 357:6 (2005), 2509-2526. MR 2006d:22027 Zbl 1065.22014

[Tauvel and Yu 2005] P. Tauvel and R. W. T. Yu, Lie algebras and algebraic groups, Springer, Berlin, 2005. MR 2006c:17001 Zbl 1068.17001

[Trimble 1994] C. E. Trimble, "Finite ring sums from $p$-adic $K$-Bessel functions", pp. 369-378 in Finite fields: theory, applications, and algorithms: Proceedings of the Second International Conference (Las Vegas, NV, 1993), edited by G. L. Mullen and P. J.-S. Shiue, Contemp. Math. 168, Amer. Math. Soc., Providence, RI, 1994. MR 96a:11134 Zbl 0835.11044

[Waldspurger 1995] J.-L. Waldspurger, "Une formule des traces locale pour les algèbres de Lie padiques”, J. Reine Angew. Math. 465 (1995), 41-99. MR 96i:22039 Zbl 0829.11030

[Yu 2001] J.-K. Yu, "Construction of tame supercuspidal representations", J. Amer. Math. Soc. 14:3 (2001), 579-622. MR 2002f:22033 Zbl 0971.22012 
Received October 7, 2010.

LOREN SPICE

DEPARTMENT OF MATHEMATICS

TEXas Christian University

TCU BOX 298900

2840 W. BowIE ST

FORT WORTH, TX 76109

UNITED STATES

1.spice@tcu.edu

http://faculty.tcu.edu/lspice 


\title{
PACIFIC JOURNAL OF MATHEMATICS
}

\author{
http://pacificmath.org \\ Founded in 1951 by \\ E. F. Beckenbach (1906-1982) and F. Wolf (1904-1989)
}

\section{EDITORS}

V. S. Varadarajan (Managing Editor)

Department of Mathematics

University of California

Los Angeles, CA 90095-1555

pacific@math.ucla.edu

Vyjayanthi Chari

Department of Mathematics

University of California

Riverside, CA 92521-0135

chari@math.ucr.edu

\section{Robert Finn}

Department of Mathematics Stanford University

Stanford, CA 94305-2125

finn@math.stanford.edu

Kefeng Liu

Department of Mathematics

University of California

Los Angeles, CA 90095-1555

liu@math.ucla.edu
Darren Long

Department of Mathematics

University of California

Santa Barbara, CA 93106-3080

long@math.ucsb.edu

Jiang-Hua Lu

Department of Mathematics

The University of Hong Kong

Pokfulam Rd., Hong Kong jhlu@maths.hku.hk

Alexander Merkurjev

Department of Mathematics

University of California

Los Angeles, CA 90095-1555

merkurev@math.ucla.edu
Sorin Popa

Department of Mathematics University of California

Los Angeles, CA 90095-1555 popa@math.ucla.edu

Jie Qing

Department of Mathematics

University of California

Santa Cruz, CA 95064

qing@cats.ucsc.edu

Jonathan Rogawski

Department of Mathematics

University of California

Los Angeles, CA 90095-1555

jonr@math.ucla.edu

\section{PRODUCTION}

pacific@math.berkeley.edu

\section{SUPPORTING INSTITUTIONS}

ACADEMIA SINICA, TAIPEI

CALIFORNIA INST. OF TECHNOLOGY INST. DE MATEMÁTICA PURA E APLICADA KEIO UNIVERSITY

MATH. SCIENCES RESEARCH INSTITUTE NEW MEXICO STATE UNIV.

OREGON STATE UNIV.

\author{
STANFORD UNIVERSITY \\ UNIV. OF BRITISH COLUMBIA \\ UNIV. OF CALIFORNIA, BERKELEY \\ UNIV. OF CALIFORNIA, DAVIS \\ UNIV. OF CALIFORNIA, LOS ANGELES \\ UNIV. OF CALIFORNIA, RIVERSIDE \\ UNIV. OF CALIFORNIA, SAN DIEGO \\ UNIV. OF CALIF., SANTA BARBARA
}

\author{
UNIV. OF CALIF., SANTA CRUZ \\ UNIV. OF MONTANA \\ UNIV. OF OREGON \\ UNIV. OF SOUTHERN CALIFORNIA \\ UNIV. OF UTAH \\ UNIV. OF WASHINGTON \\ WASHINGTON STATE UNIVERSITY
}

These supporting institutions contribute to the cost of publication of this Journal, but they are not owners or publishers and have no responsibility for its contents or policies.

See inside back cover or pacificmath.org for submission instructions.

The subscription price for 2011 is US \$420/year for the electronic version, and \$485/year for print and electronic.

Subscriptions, requests for back issues from the last three years and changes of subscribers address should be sent to Pacific Journal of Mathematics, P.O. Box 4163, Berkeley, CA 94704-0163, U.S.A. Prior back issues are obtainable from Periodicals Service Company, 11 Main Street, Germantown, NY 12526-5635. The Pacific Journal of Mathematics is indexed by Mathematical Reviews, Zentralblatt MATH, PASCAL CNRS Index, Referativnyi Zhurnal, Current Mathematical Publications and the Science Citation Index.

The Pacific Journal of Mathematics (ISSN 0030-8730) at the University of California, c/o Department of Mathematics, 969 Evans Hall, Berkeley, CA 94720-3840, is published monthly except July and August. Periodical rate postage paid at Berkeley, CA 94704, and additional mailing offices. POSTMASTER: send address changes to Pacific Journal of Mathematics, P.O. Box 4163, Berkeley, CA 94704-0163.

PJM peer review and production are managed by EditFLOW ${ }^{\mathrm{TM}}$ from Mathematical Sciences Publishers.

PUBLISHED BY PACIFIC JOURNAL OF MATHEMATICS

at the University of California, Berkeley 94720-3840

A NON-PROFIT CORPORATION

Typeset in LATEX

Copyright $(2011$ by Pacific Journal of Mathematics 


\section{PACIFIC JOURNAL OF MATHEMATICS}

Volume $254 \quad$ No. $2 \quad$ December 2011

Curvatures of spheres in Hilbert geometry

ALEXANDER BORISENKO and EUGENE OLIN

A formula equating open and closed Gromov-Witten invariants and its applications 275

to mirror symmetry

KWOKWAI CHAN

A note on $p$-harmonic $l$-forms on complete manifolds

LiAng-CHu CHANG and CHIUng-JUe ANNA SUNG

The Cheeger constant of curved strips

DAVID KREJČIŘíK and ALdo PRATELLI

Structure of solutions of 3D axisymmetric Navier-Stokes equations near maximal points

\section{ZHEN LEI and QI S. ZHANG}

Local comparison theorems for Kähler manifolds

GANG LIU

Structurable algebras of skew-rank 1 over the affine plane

SUSANNE PUMPLÜN

An analogue of Krein's theorem for semisimple Lie groups

SANJOY PUSTI

Une remarque de dynamique sur les variétés semi-abéliennes

GAËL RÉMOND

Fourier transforms of semisimple orbital integrals on the Lie algebra of $\mathrm{SL}_{2}$

LOREN SPICE

On noncompact $\tau$-quasi-Einstein metrics

LIN FENG WANG

Decomposition of de Rham complexes with smooth horizontal coefficients for semistable reductions

QIHONG XIE

A differentiable sphere theorem inspired by rigidity of minimal submanifolds 